Check for updates

Cite this: J. Mater. Chem. A, 2020, 8 , 21526

Received 15th August 2020

Accepted 22nd September 2020

DOI: $10.1039 / \mathrm{d0ta0} 8009 \mathrm{~h}$

rsc.li/materials-a

\section{Synthesis, characterization and application of defective metal-organic frameworks: current status and perspectives}

\begin{abstract}
Wenlong Xiang, ${ }^{a}$ Yueping Zhang, ${ }^{\mathrm{b}}$ Yifei Chen, ${ }^{\star c}$ Chang-jun Liu (D) *a and Xin Tu (D) *d
Defects are now recognized to be a useful tool in tailoring the properties of metal-organic frameworks (MOFs). The introduction of missing linker and cluster defects into MOFs provides additional active sites, optimizes the acidity/basicity, improves the conductivity, tailors mechanical responses and creates more pore space to enhance diffusion and mass transfer in MOFs. Structural defects in MOFs have been demonstrated to be beneficial in areas such as catalysis, decontamination, bio-applications, adsorption, separation, energy storage, energy conversion, electronics, magnetics, optical functional materials and others. Defective MOFs are also excellent model materials for the fundamental study of defect chemistry. In this review, we provide a general overview of the commonly available methods that are feasible for the creation and characterization of structural defects in MOF materials. Additionally, recent studies on various applications of defective MOFs are highlighted, aiming to provide new insights into the design and introduction of structural defects to synthesize MOF materials with high performance and to promote the wide application of defective MOFs in various fields. Challenges and future perspectives on defect engineering of MOFs are also addressed.
\end{abstract}

${ }^{a}$ Collaborative Innovation Center of Chemical Science and Engineering, School of Chemical Engineering and Technology, Tianjin University, Tianjin, 300072, China. E-mail: ughg_cjl@yahoo.com

${ }^{b}$ Department of Chemistry, Tianjin University, Tianjin, 300072, China

${ }^{c} R \& D$ Center for Petrochemical Technology, Tianjin University, Tianjin 300072, China. E-mail: tdchenyifei@126.com

${ }^{d}$ Department of Electrical Engineering and Electronics, University of Liverpool, Liverpool L69 3GJ, UK. E-mail: xin.tu@liverpool.ac.uk

\section{Introduction}

Metal-organic frameworks (MOFs), unique porous crystalline materials assembled by the coordination bonds of inorganic metal ions with organic ligands, have received remarkable attention over the past decade due to their designable porosity, surface chemistry, and the functionalization of the coordination space. ${ }^{1-3}$ There is an increasing interest in the defect engineering of MOFs. Defects have now been recognized to be

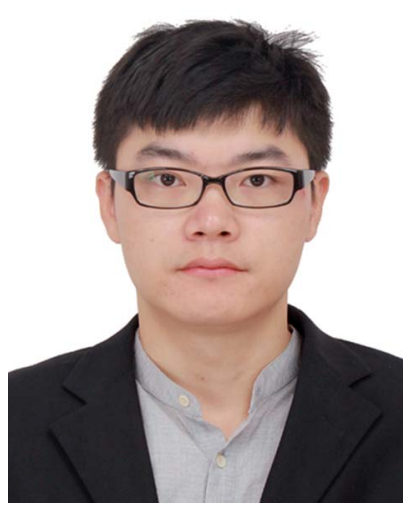

Wenlong Xiang is currently a Ph.D. candidate in the School of Chemical Engineering and Technology at Tianjin University, China. He received his B.S. degree in 2015 from China University of Mining and Technology (Beijing). His research topics are metal-organic framework based materials for $\mathrm{CO}_{2}$ chemical transformations and the desulfurization of gaseous hydrocarbons. He has published four papers in Applied Energy, Microporous and Mesoporous Materials, Catalysis Today and Molecules, covering defective metal-organic frameworks for $\mathrm{CO}_{2}$ conversion.

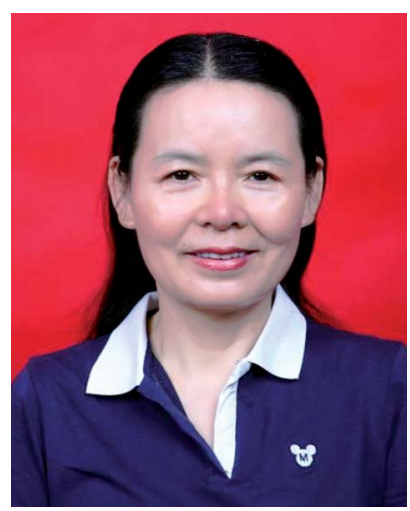

Yueping Zhang is an Associate Professor of Tianjin University. She received her $P h D$ degree from Tianjin University in 2004. Her research interests include plasma chemistry, C1 chemistry, and preparation and characterization of catalysts. 
b

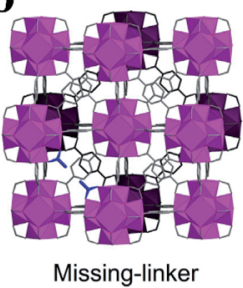

a

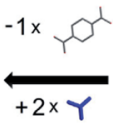

$+2 \times Y$

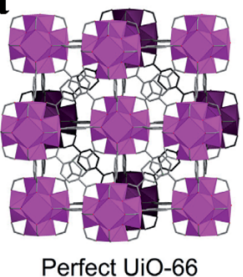

c

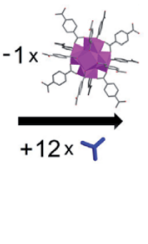

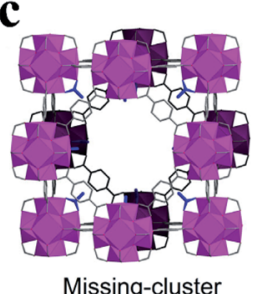

Fig. 1 Illustration of the structural differences between the ideal UiO-66 unit cell and those with missing cluster/missing linker defects. Reproduced with permission. ${ }^{43}$ Copyright 2017, Elsevier.

a useful tool to tailor the physical-chemical properties of MOFs, such as adsorption capacity, ${ }^{4-12}$ band structures, ${ }^{13-15}$ conductivity ${ }^{16,17}$ mechanical responses, ${ }^{18,19}$ and catalytic properties. ${ }^{20-40}$ Until now, both experimental and theoretical calculation studies have demonstrated that a certain degree of structural defects can offer positive effects on the physical and chemical properties of MOF materials. ${ }^{\mathbf{4 1 - 4 4}}$ However, it is also noted that

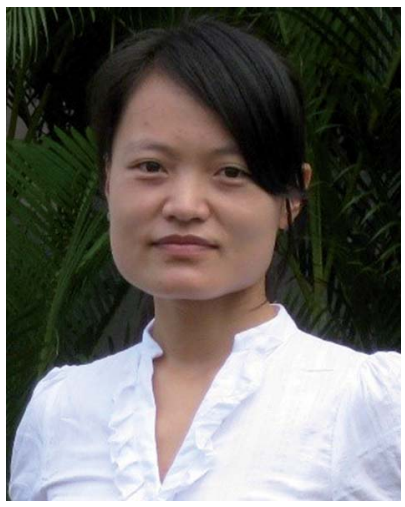

Yifei Chen is an AssociateProfessor of Tianjin University. She received her $P h D$ degree from the National University of Singapore in 2012 with the supervisor of Prof. Jianwen Jiang. Her major research interests are the design and synthesis of metal-organic frameworks for application in separation and catalysis. She has published over 60 papers on this topic in journals like ChemSusChem, Langmuir, Journal of Chemical Physics, Physical Chemistry Chemical Physics and ACS Applied Materials \& Interfaces.

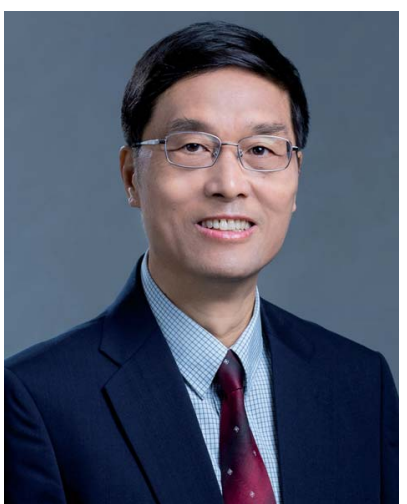

Chang-jun Liu is a Chang Jiang Distinguished Professor and Fellow of the Royal Society of Chemistry. His research interests include $\mathrm{CO}_{2}$ utilization, natural gas conversion, plasma nanoscience, and $3 D$ printing. He has been in the list of highly cited Chinese authors (Chemical Engineering) by Elsevier since 2014. He served as 2010 Program Chair of Fuel Chemistry Division of American Chemical Society and Chair of $10^{\text {th }}$ International Conference on $\mathrm{CO}_{2}$ Utilization. He is in the advisory board of Greenhouse Gases: Science \& Technology and in the editorial board of Applied Catalysis B, Journal of $\mathrm{CO}_{2}$ Utilization and Chinese Journal of Catalysis. defects sometimes result in negative effects on some MOF properties such as gas adsorption and diffusion as well as optical properties. ${ }^{45}$ In addition, structural defects are often associated with a decreased chemical, thermal, and mechanical stability of framework structures. Therefore, the framework must take into account its stability and provide tolerance to such defects. Wang et al. ${ }^{45}$ summarized several efficient strategies for overcoming defects of HKUST-1. How to fully use the specific properties of MOF materials endowed by structural defects is critical for practical applications.

Defects in MOFs have been defined as "sites that locally break the regular periodic arrangement of atoms or ions of the static crystalline parent framework because of missing or dislocated atoms or ions". ${ }^{46}$ Generally, defects can be classified into point, line, and planar defects on the geometric scale. Within the field of MOFs, point defects have been subjected to intense focus and have been widely investigated in various fields due to their important influence on the properties of MOFs. ${ }^{42,43,47}$ The most important two types of point defects in MOF materials are missing-linker defects and missing-cluster defects (Fig. 1). In the case of zirconium-based MOFs, missing-linker defects are generated when an organic linker is removed from the structure, leaving coordination vacancies on two adjacent metal clusters (Fig. 1a to b), whereas missing-

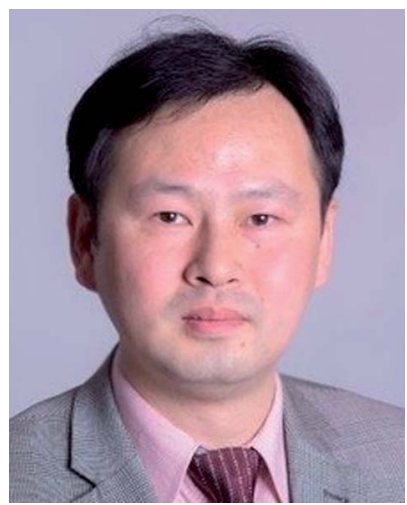

Xin $T u$ received a dual $P h D$ degree from the University of Rouen (France) and Zhejiang University (China) in 2007. He is Chair Professor of Plasma Catalysis in the Department of Electrical Engineering and Electronics at the University of Liverpool. His current research interests include low temperature plasma-catalysis for gas clean-up, C1 chemistry (e.g., $\mathrm{CO}_{2}$ conversion and $\mathrm{CH}_{4}$ activation), nitrogen fixation and biomass conversion, and the use of cold plasma for the synthesis of catalysts and carbon materials. 
cluster defects are the result of the removal of a $\left[\mathrm{Zr}_{6} \mathrm{O}_{4}(\mathrm{OH})_{4}\right]^{12+}$ cluster together with its entire set of twelve organic linkers, generating one coordination vacancy on each of the neighboring clusters (Fig. 1a to c). Essentially, missing-cluster defects are a result of the critical concentration of missing-linker defects and their spatial distribution. ${ }^{47}$ However, it is noted that the equilibrium between these two types of point defects, missing-linker and missing-cluster defects, is still not clear. In most cases, missing-linker and missing-cluster defects are only indirectly accessible through conventional methods. Recently, structural defects in UiO-66 (Universitetet i Oslo 66, $\mathrm{Zr}_{6}(\mathrm{OH})_{4}{ }^{-}$ $\mathrm{O}_{4}(\mathrm{BDC})_{6}, \mathrm{BDC}=$ benzene-1,4-dicarboxylate) have been imaged at a sub-unit-cell resolution. ${ }^{48}$ This work provides direct evidence to support the existence of missing linker and missing cluster defects in MOFs. Many MOFs, even the most carefully synthesized MOFs, are known to contain structural defects. Although defect engineering in MOFs is still in its infancy, a significant effort has been devoted to detecting and controlling defects. ${ }^{\mathbf{4}}$ There has been a growing interest in understanding the formation of these defects and exploiting their various practical applications. ${ }^{41}$ It is expected that defect engineering in MOFs will continue to move forward in the coming years with the development of advanced characterization technologies and the further understanding of defect chemistry in MOFs.

In this review, we give a general overview of commonly available approaches and new developments for the preparation of defective MOFs. The current state-of-the-art in the characterization of structural defects in MOFs is summarized. Important advances in the use of defective MOFs for adsorption, catalysis, decontamination, biological medicine and smart applications are discussed. Challenges and future developments in the emerging area are addressed as well.

\section{Formation of defects in MOFs}

Methods developed and applied for the formation of defects in MOFs have been well summarized in previous reviews. ${ }^{41,42,47}$ The reported methods can be divided into two synthetic approaches, known as the "de novo" synthesis and the post-synthetic treatment approach. A schematic representation of the mainly applied methods to introduce defects in MOFs is shown in Fig. 2.

\subsection{De novo synthesis}

Judicious choices of synthetic conditions have been demonstrated to effectively promote the generation of defects in the de novo synthesis of MOF materials. Among these choices, the most common way is the addition of the so-called modulators (e.g., monocarboxylic acids) to the linker molecules during the synthesis of MOFs. ${ }^{47}$ A number of key factors have been considered for the generation of defects in the synthesis of MOFs, including solvent, the type of modulators, the number of modulators and the crystal growth rate. ${ }^{\mathbf{4 2 4 9}}$ To date, several typical monocarboxylic modulators, including formic acid (FA), ${ }^{6,50}$ acetic acid, ${ }^{23,25,27}$ difluoroacetic acid (DFA), ${ }^{51}$

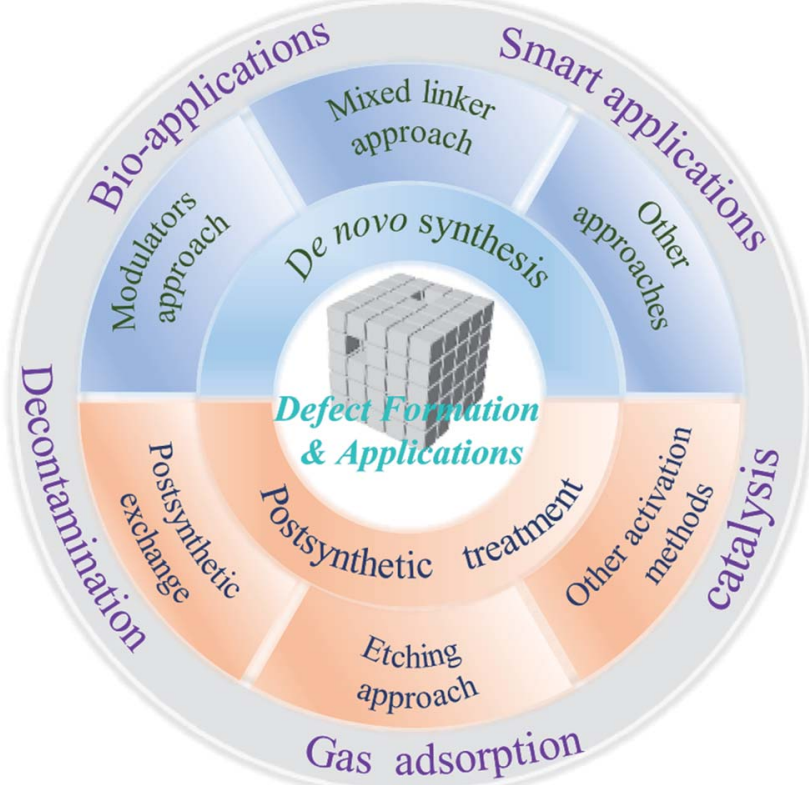

Fig. 2 A representation of the main procedures used to create defects and a variety of applications for structural defects in MOFs.

trifluoroacetic acid (TFA), ${ }^{29}$ benzoic acid (BA), ${ }^{7}$ and others ${ }^{33,52,53}$ have been applied in the synthesis of defective MOFs. Shearer et $a .^{51}$ systematically investigated the effect of modulators (acetic acid, FA, TFA, and DFA) on the defect chemistry of UiO66. They found a correlation between the defect concentration and the Brønsted acidity of the modulator (Fig. 3a). The densities of the missing-linker or missing-cluster defects can be tuned by altering the acidity of the modulator ${ }^{51}$ or the concentration of the modulator. ${ }^{23,33}$ Besides monocarboxylic modulators, nitrogenous heterocyclic modulators (e.g., 1methylimidazole) have also been recently reported. ${ }^{28}$ The modulation of the temperature also plays an important role in the de novo synthesis of defective MOFs. The combination of the modulator acetic acid and thermal modulation to tune the densities of defect sites in UiO-66 was reported. ${ }^{54}$ Changing the synthesis temperature can control the number of defect sites in MOFs with a maximal number of defect sites (around 1.3 missing linkers per $\mathrm{Zr}_{6}$ node) being achieved at a temperature of $45{ }^{\circ} \mathrm{C}$ (Fig. 3b).

Recently, Jiang et $a .^{53}$ reported a versatile modulatorinduced defect-formation strategy for the rational design and controllable synthesis of hierarchically porous MOFs (HPMOFs) with high stability. The excessive modulator alkyl monocarboxylic acid coordinates to metal ions for the formation of metal-oxo clusters, while the alkyl chain creates structural defects and additional pore space (Fig. 3c). Moreover, the pore space can be tuned via altering the length and concentration of the modulator (Fig. 3d-f). The defect-formation strategy to create hierarchical pores in MOF materials has been summarized in previous reviews. ${ }^{55,56}$ Additionally, previously reported studies demonstrated that modulator-induced missing linker defects can be successfully eliminated via 

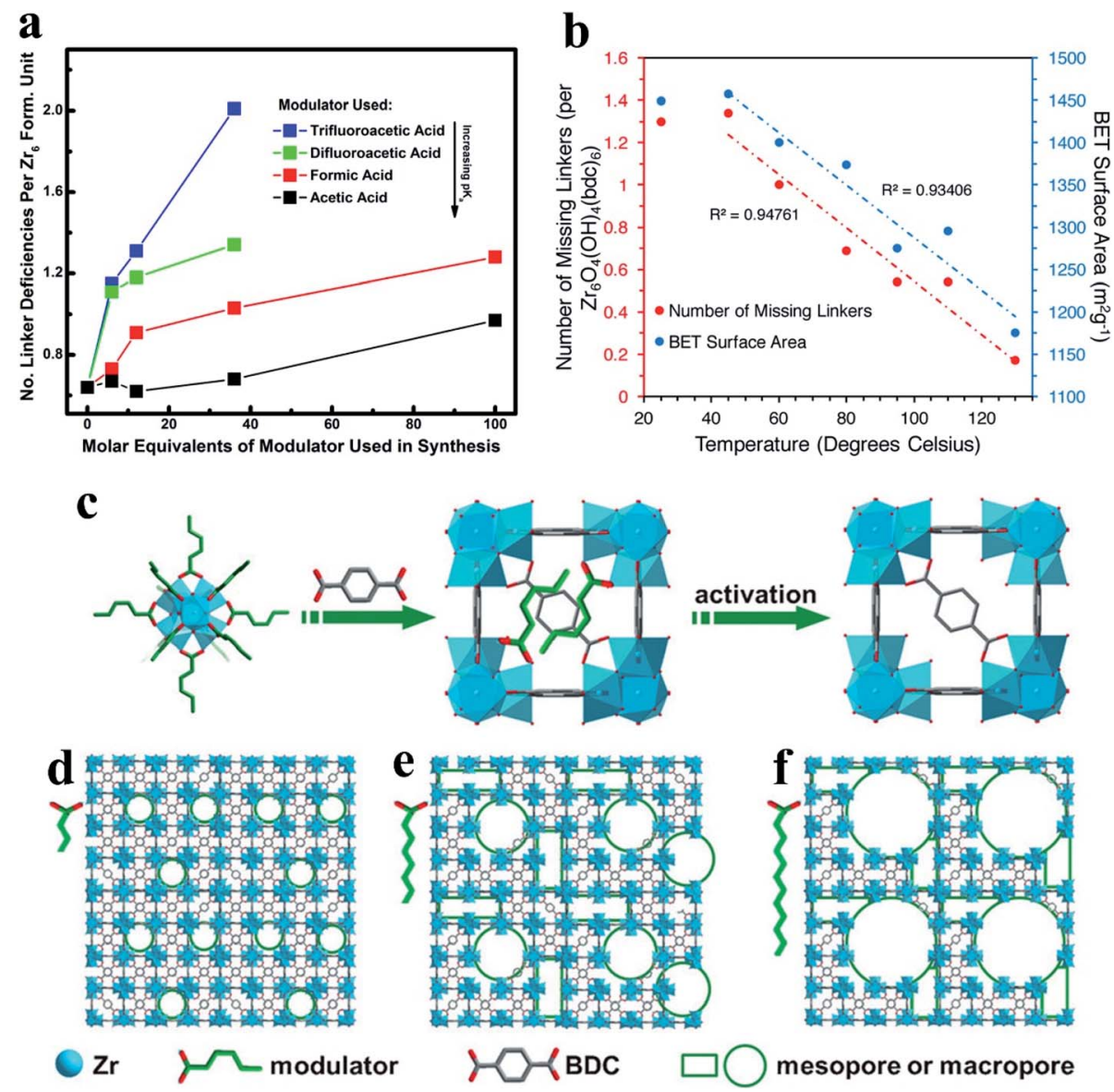

Fig. 3 (a) A plot showing the number of linker deficiencies per $\mathrm{Zr}_{6}$ formula unit against the molar equivalents of the modulator in UiO-66. Reproduced with permission. ${ }^{51}$ Copyright 2016, American Chemical Society. (b) Correlations between the synthesis temperature and the number of missing linkers per $\mathrm{Zr}_{6}$ unit (red). Reproduced with permission. ${ }^{54}$ Copyright 2017, American Chemical Society. (c-f) Schematic illustrations of the synthesis of HP-MOFs with adjustable porosity using UiO-66 as an example. Reproduced with permission..$^{53} \mathrm{Copyright} 2017$, Wiley-VCH.

linker exchange. ${ }^{57,58}$ In addition to the synthesis temperature and modulators, water has also been confirmed to play an important role in the synthesis of defect phases of UiO family MOFs. ${ }^{59}$ Previous work showed that water and hydroxide can introduce missing-linker defects into the framework by replacing the bidentate dicarboxylate linkers. ${ }^{59}$

In addition, the mixed-linker approach represents another efficient strategy to create defects in MOF structures. Larger linkers with different coordinating groups can partially substitute linkers from a parent framework in de novo synthesis. The mixedlinker approach can increase the number of defect sites or active sites by incorporating another linker with various unique functionalities..$^{34,60-63}$ By using this mixed-linker approach, Epp et al. ${ }^{61}$ successfully introduced point defects at the distinct $\mathrm{Ru}_{2}$ paddlewheel nodes in ruthenium MOF Ru-HKUST-1. Mixtures of benzene-1,3,5-tricarboxylate and pyridine-3,5-dicarboxylate linkers were used in the mixed-linker approach. Similarly, tetrakis(4-carboxyphenyl)porphyrin (TCPP) was used as a functional secondary ligand and integrated into stable UiO-66 via an in situ one-pot synthetic method. ${ }^{62}$ The authors found that TCPP occupied some coordination sites of the $\mathrm{Zr}_{6}$ clusters, leading to the generation of defects in the UiO-66 framework.
Recently, a powerful strategy combining the mixed-linker approach and the labile linker decomposition has been developed to create structural defects and hierarchical pores in MOFs. ${ }^{64,65}$ Stable linkers and labile linkers are firstly incorporated into the MOF structure via a mixed-linker approach, followed by the post-synthetic decomposition of labile linkers. As a result of the decomposition of labile linkers, rich missinglinker defects were formed (Fig. 4a). To date, hydrolysis, ${ }^{64}$ thermolysis, ${ }^{11,65,66}$ ozonolysis, ${ }^{67}$ and laser photolysis ${ }^{68}$ have been reported to eliminate labile linkers selectively. Zhou et al. ${ }^{66}$ demonstrated the versatility of labile linker decomposition by extending the strategy to common linkers and different metal clusters. As shown in Fig. 4b, the combination of two suitable linkers (thermolabile and thermostable linkers) can form the corresponding sets of multivariate metal-organic frameworks (MTV-MOFs). Furthermore, MTV-MOFs with different metal clusters (e.g., MOF-5(Zn), MIL-53(Fe), MIL-125(Ti), and UiO66(Hf)) have been successfully synthesized. By selectively removing thermolabile linkers, all sets of MTV-MOFs can be transformed from microporosity to hierarchical porosity without the loss of crystallinity. Missing linker defects and even missing cluster defects were generated in MTV-MOFs 

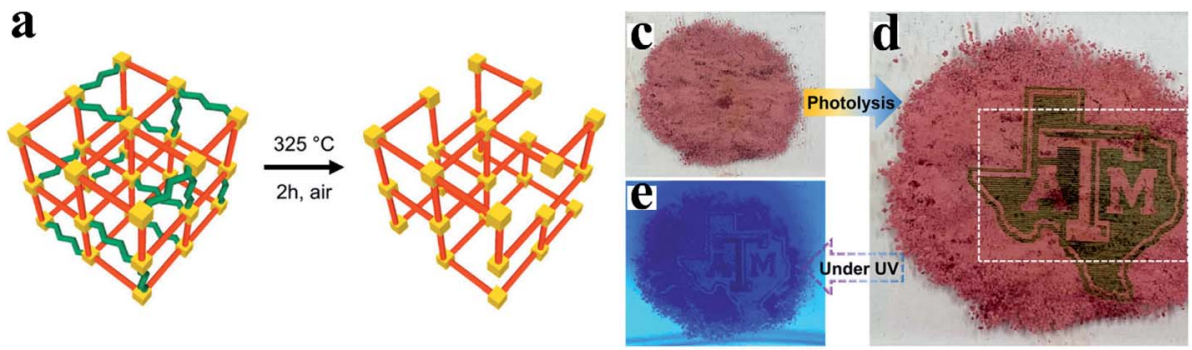

b

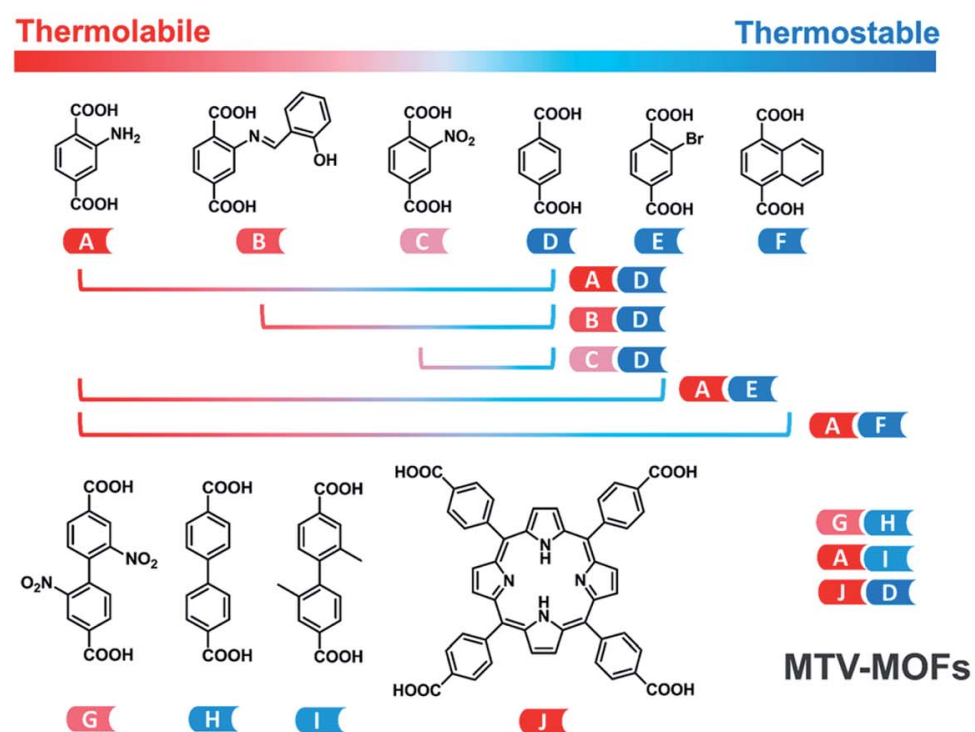

Fig. 4 (a) Defect creation by decomposing the labile linkers (green) in the stable (red) matrix. Yellow: $\mathrm{Zr}_{6}$ nodes. Reproduced with permission. ${ }^{65}$ Copyright 2017, American Chemical Society. (b) The versatility of controllable linker thermolysis to construct HP-MOFs with various linkers. Reproduced with permission. ${ }^{66}$ Copyright 2018, American Chemical Society. Optical photographs of (c) the original UiO-66-TCPP-5\% powder, (d) a logo on the UiO-66-TCPP-5\% powder after programmable photolithography and (e) the patterned MOF sample under a UV lamp. Adapted with permission. ${ }^{68}$ Copyright 2020, Wiley-VCH.

structures. Note that thermal treatment parameters including temperature and treatment time should be chosen cautiously to avoid the complete collapse of the framework. Importantly, Zhou $e t ~ a l .{ }^{68}$ further developed a laser photolytic process for the removal of the photolabile linker (TCPP), creating mesopores within microporous MOF UiO-66 in tens of milliseconds. The laser photolysis precisely controls the size and spatial arrangement of hierarchical pores, while ensuring the stability and integrity of the original framework. Complicated patterns on MOFs can be fabricated in a highly controllable manner using this laser photolysis strategy (Fig. 4c-e).

Additionally, novel de novo synthesis methods have been recently reported to introduce defects in MOF materials. ${ }^{12,30,32}$ $\mathrm{Hu} e t \mathrm{al}^{30}$ used a microfluidic laminar flow for the synthesis of enzyme-embedded defect-rich MOFs, which are difficult to achieve using a conventional bulk solution synthesis (Fig. 5a and b). Under a continuously changing concentration of MOF precursors in the gradient mixing on-chip (Fig. 5c), the resulting products showed structural defects and therefore, mesopores. The defect property (like the pore width and pore volume) can be controlled by adjusting the flow rate of the reactants into the microchannel. Similarly, templated electrosynthesis has been recently reported with the ability to integrate mesopores and crystal defects into MOFs (Fig. 5d). ${ }^{32}$ An ionic liquid was used as both an electrolyte and template in the templated electrosynthesis. The mesoporous Cu(II)-MOF MFM-100 with crystal defects was successfully obtained via self-aggregation. The formation of crystal defects with uncoupled $\mathrm{Cu}$ (II) centers can be evidenced by confocal fluorescence microscopy (CFM) analysis (Fig. 5e and f).

\subsection{Post-synthetic treatment}

In addition to the "de novo" synthetic approaches to introduce structural defects into MOFs, another synthetic approach toward defective MOFs is post-synthetic treatment. ${ }^{69}$ Postsynthetic treatment involves a heterogeneous treatment of the parent MOFs with modified linkers, ${ }^{70}$ etching agents, ${ }^{36,71}$ or other harsh activation procedures (e.g., thermal activation ${ }^{72,73}$ and plasma activation ${ }^{74}$ ).

The most established post-synthetic treatment method is post-synthetic exchange (PSE), involving the metathesis of metal ions or ligands from intact MOFs..$^{58,70,75}$ The metal ion exchange process involves a substitution of original metal ions with other metal ions that have a different coordination number compared to the original metal ions in MOFs. ${ }^{41,76}$ The post- 


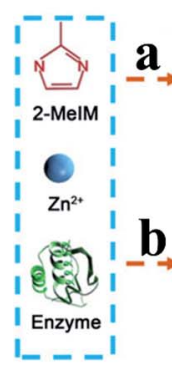

c

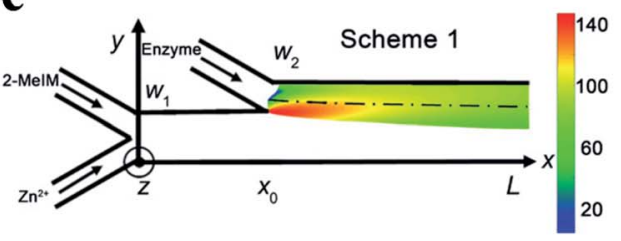

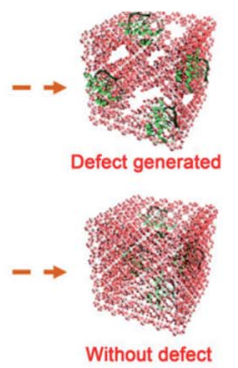

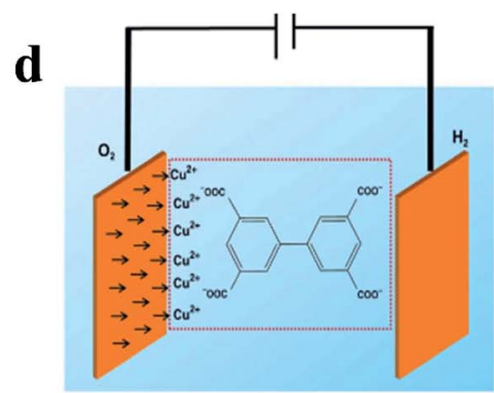

e
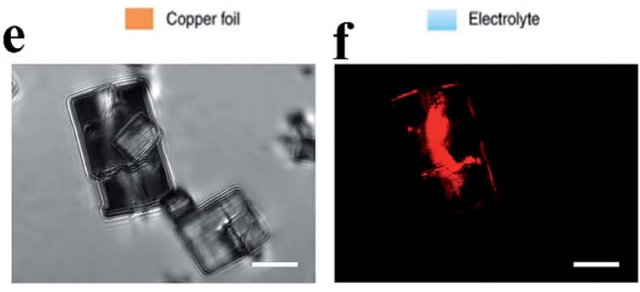

Fig. 5 Schematics of (a) microfluidic laminar flow synthesis and (b) bulk solution synthesis of enzyme-MOF composites. (c) A simulated heat map of the molar ratio between 2-MelM and $\mathrm{Zn}^{2+}$ alongside the microchannel. Adapted with permission. ${ }^{30}$ Copyright 2020 , American Association for the Advancement of Science. (d) A schematic diagram of the electrosynthesis of MFM-100. (e) Micrographs and (f) CFM images for MFM-100a. The scale bar is $5 \mu \mathrm{m}$ in all images. The fluorescence (shown in red) indicates the presence of crystal defects. Reproduced with permission. ${ }^{32}$ Copyright 2019, Springer Nature.

synthetic exchange of ligands, also known as solvent-assisted linker exchange, can replace the parent ligands of the MOF with other modified ligands via a heterogeneous reaction using a suitable solvent. PSE can introduce diverse chemical functionalities and defect sites into existing MOF structures using modified ligands (e.g., functionalized ligands $\mathrm{s}^{77}$ or ligands with an incorporated catalyst precursor ${ }^{70}$ ). Importantly, PSE provides a new route for the synthesis of novel MOF materials that are difficult to prepare through other traditional synthetic methods. ${ }^{77}$

Another common route in post-synthetic treatment for defect generation in MOFs involves etching agents (e.g., acid, base, and salts)..$^{8,36,71,78-80}$ The etching process introduces not only missing linker/cluster defects but also mesopores and even macropores in microporous MOFs. For example, Gu et al. ${ }^{79}$ reported a simple and effective acid etching strategy to introduce structural defects and mesopores into UiO-66. Propionic acid partially replaces the bridging ligands in UiO-66 and leads to the departure of partial ligands and metal clusters. Similarly, potassium hydroxide leads to the formation of missing-linker defects and the presence of extra-framework cations in nickel pyrazolate MOFs. ${ }^{8}$ Chang et $a l .{ }^{36}$ used tetrafluoroborate as both functional sites and etching agents to create defects and mesopores in microporous Al-MOF. A possible mechanism to illustrate the formation of defect-mesopores is shown in Fig. 6a. The loading of copper(II) tetrafluoroborate cleaves the existing chemical bonds between $\mathrm{Al}^{3+}$ and the carboxyl group to generate defect-induced mesopores. The mesopore size and active sites can be precisely tuned by carefully controlling the loading amount of the salt etching agent. A low concentration of salt only resulted in a relatively mild etching of the crystal structure, whilst a high concentration loading could induce a deep etching of the framework (Fig. 6a).
Recently, plasma etching, a green and facile technology, has been developed to generate defects in MOF materials. ${ }^{21,44,74,81}$ Plasma contains highly reactive species (e.g., electrons, ions and free radicals), but the bulk gas temperature of the plasma remains near room temperature during the plasma treatment. ${ }^{82}$ The combined advantages of high reactivity and limited thermal effect have made plasma technology promising in the creation of defects in MOFs without damaging their framework structures. Yu et al. ${ }^{44}$ used an ionized nitrogen plasma to successfully break the bonds of iron-carbon-nitrogen-nickel units in nickel-iron Prussian blue analogues, creating unconventional carbon-nitrogen vacancies (Fig. 6b). This work opens up new opportunities for producing vacancy defects in nanomaterials. Recently, we used argon plasma for the rapid introduction of missing linker defects in UiO-66 at ambient pressure and low temperature (less than $125^{\circ} \mathrm{C}$ ) without damaging its bulk structure. ${ }^{81}$ The energetic and reactive species generated in the argon plasma decompose some of the linkers within UiO-66 structures, creating rich coordinatively unsaturated metal sites. Tunable defect densities in defective UiO-66 can be achieved by controlling the plasma treatment time. The number of linker deficiencies per $\mathrm{Zr}_{6}$ cluster can reach up to 2.3 after an argon plasma treatment of 30 minutes.

\section{Characterization of defective MOFs}

MOFs provide an excellent platform for the study of defect chemistry because of their diverse and clear chemical structures. The characterization of defects, such as the defect concentration and spatial distribution, remains a significant challenge. Several techniques and their combinations have been employed to detect structural defects in MOFs, such as powder X-ray diffraction (PXRD), ${ }^{48,51,83}$ thermogravimetric 

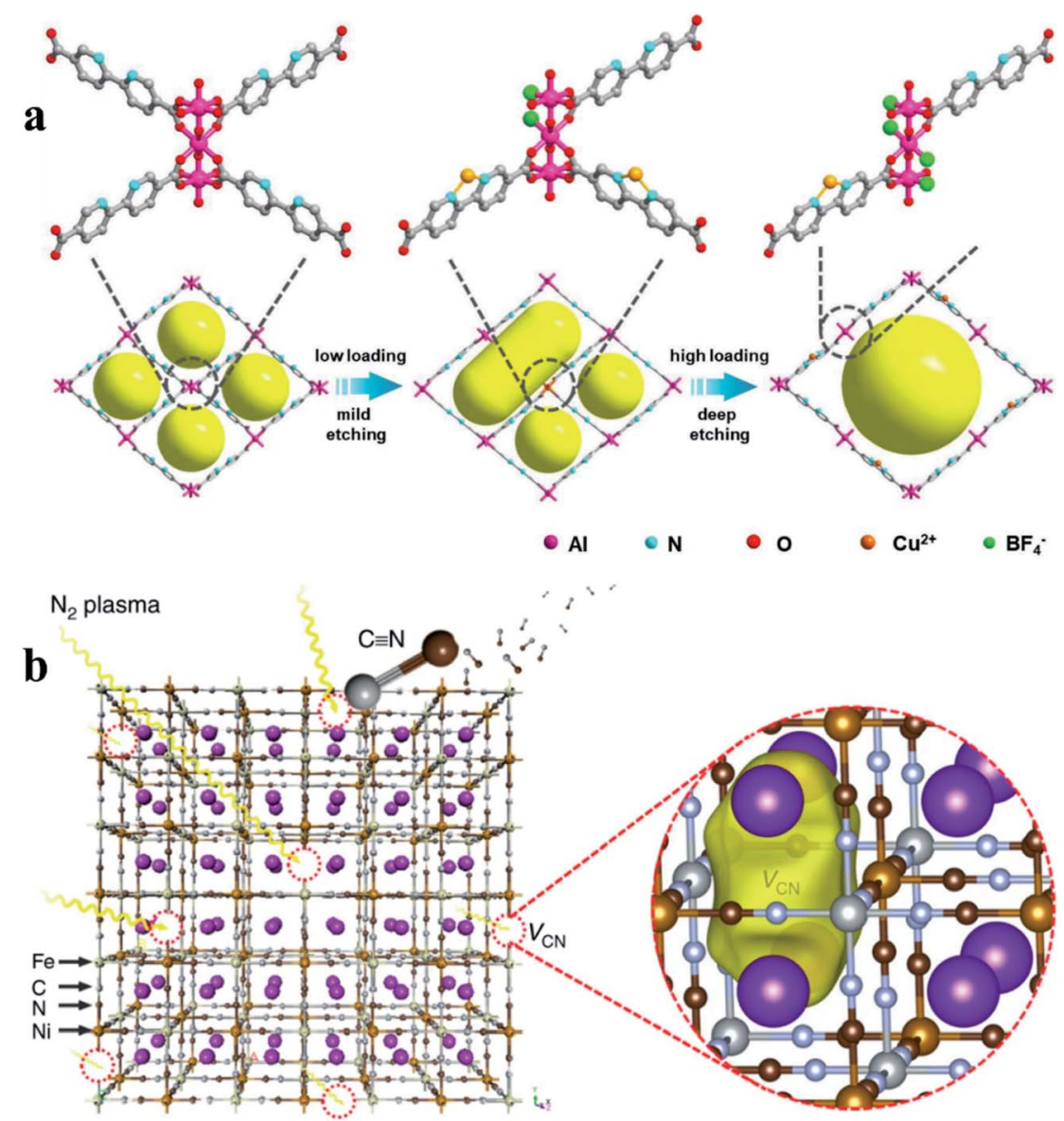

Fig. 6 (a) An illustration showing the creation of structural defects in Al-bpydc structures. Reproduced with permission. ${ }^{36}$ Copyright 2019 , Wiley$\mathrm{VCH}$. (b) A schematic illustration of the preparation of $\mathrm{V}_{\mathrm{CN}}-$ mediated Ni-Fe PBA. $\mathrm{V}_{\mathrm{CN}}$ forms in the Ni-Fe PBA material using $\mathrm{N}_{2}$ plasma bombardment. Reproduced with permission. ${ }^{44}$ Copyright 2019, Springer Nature.

analysis (TGA), ${ }^{29,83,84}$ acid-base titration, ${ }^{22,54,85,86}$ water sorption, ${ }^{87,88}$ high-resolution nuclear magnetic resonance (NMR), ${ }^{5,9,57,89}$ electron paramagnetic resonance (EPR), ${ }^{11,37,90,91}$ positron annihilation lifetime spectroscopy (PALS), ${ }^{\mathbf{9 2}-94}$ and extended X-ray absorption fine structure (EXAFS). ${ }^{33,90,95}$ These characterization techniques can be generally divided into two groups: spectroscopic and microscopic methods.

XRD analysis has been used to qualitatively examine the ordered defective phase of Zr-MOFs. ${ }^{96}$ Broad peaks associated with the formation of ordered missing-cluster defects were observed in the low angle region. ${ }^{29,83,97}$

EPR can detect unpaired electrons in paramagnetic species and is a powerful technique to detect oxygen vacancies. ${ }^{98}$ In a MOF where the organic linkers are carboxylic acid species, the presence of missing linker defects generates oxygen vacancies or coordinatively unsaturated metal defects. EPR has now been widely employed to detect missing linker defects in MOFs. ${ }^{\mathbf{1 1 , 9 0}}$ For example, Xu et al. ${ }^{11}$ used the EPR technique to evaluate the concentration of oxygen vacancies in hierarchically porous UiO66 (HP-UiO-66). The authors reported that the concentration of oxygen vacancies could represent the number of coordinatively unsaturated zirconium atoms. As shown in Fig. 7a, a symmetrical signal at $g=2.003$ in the EPR spectra of the HP-UiO-66 sample, assigned to oxygen vacancies, increases with the concentration of defects. The EPR results indicated that HPUiO-66 possesses plentiful and tunable coordinatively unsaturated zirconium atoms.

PALS, as an analytical tool, provides evidence of the inner pores in porous materials. ${ }^{92-94}$ In a MOF, the main electron density is located at the framework. As a result, the lifetime of positrons is coupled to the pore size. For example, the positron lifetime and the positron annihilation intensity were clearly enhanced for defect-rich UiO-66 compared with the near defectfree UiO-66. ${ }^{94}$ The authors found that the modulator during the synthesis of UiO-66 resulted in missing-linker defects, resulting in the expanded pore size of the framework.

EXAFS measurements can probe the local structure of MOFs..$^{33,90,95}$ Xue et al. ${ }^{33}$ reported that the Fourier transforms of EXAFS confirmed the change of local coordination geometry of $\mathrm{Co}^{2+}$ ions after the introduction of missing linker defects into Co-BDC MOF. The fitting curve revealed that the Co-O distance of defective Co-BDC (termed as CoBDC-Fc $\left.\mathrm{C}_{0.17}\right)(2.08 \AA)$ was almost identical to that of pristine CoBDC (2.07 $\AA$ ) (Fig. 7b). However, the coordination number of $\mathrm{Co}-\mathrm{O}$ for $\mathrm{CoBDC}-\mathrm{Fc}_{0.17}$ was smaller than that of CoBDC (4.4 vs. 6.2). These observations 

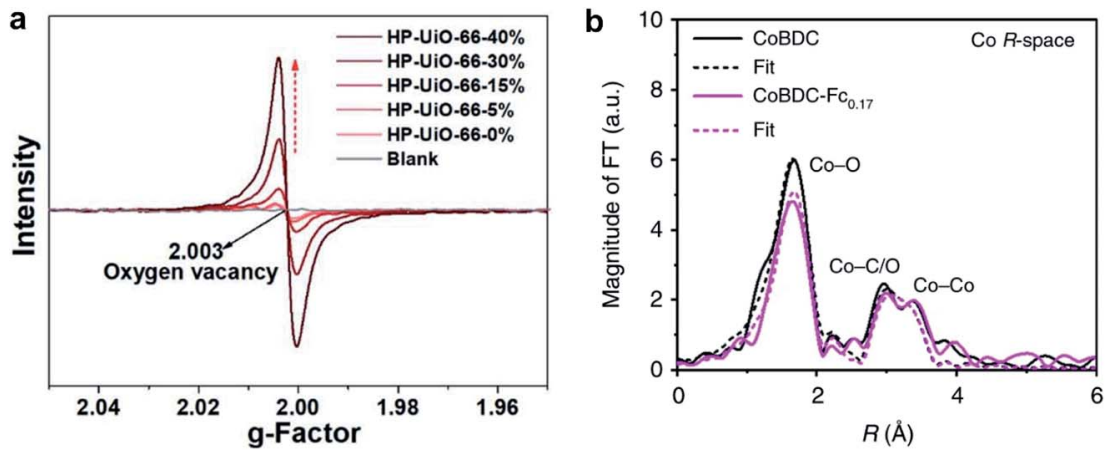

Fig. 7 (a) EPR spectra of HP-UiO-66. Reproduced with permission. ${ }^{11}$ Copyright 2020, Royal Society of Chemistry. (b) Fourier transformed EXAFS spectra of $\mathrm{COBDC}$ and $\mathrm{COBDC}-\mathrm{FC}_{0.17}$. Reproduced with permission. ${ }^{33}$ Copyright 2019, Springer Nature.

suggest that introducing missing linkers generates unsaturated $\mathrm{Co}^{2+}$ sites.

Fourier transform infrared (FTIR) analysis has been employed to verify the break of $\mathrm{C}=\mathrm{O}$ in UiO-66, ${ }^{66,83}$ which is related to missing-linker defects. In situ IR was used to monitor the crystallization process of $\mathrm{Zr}$-fumarate $\mathrm{MOF}$ in both $\mathrm{H}_{2} \mathrm{O}$ - and DMF-based systems. ${ }^{99}$ The participation of the modulator formic acid during the structure formation of the Zr-fumarate MOF was only observed in the DMF-based system, resulting in the formation of structural defects. Besides, FTIR spectra of adsorbed specific probe molecules (e.g., $\mathrm{CO},{ }^{\mathbf{1 0 0 , 1 0 1}} \mathrm{CD}_{3} \mathrm{CN},{ }^{\mathbf{8 0 , 1 0 2}}$ and pyridine $\left.{ }^{27,81,103}\right)$ have been used to characterize coordinatively unsaturated metal sites, which are associated with missing linker defects. Recently, Gentile et al. ${ }^{\mathbf{1 0 4}}$ reported a combination of the FTIR and Raman spectra of the HKUST-1 MOF with an $a b$ initio theoretical analysis of the vibrational spectra to reveal spectroscopic signatures of framework defects.

For the measurement of bulk defect concentration in MOFs, several conventional methods such as TGA, ${ }^{29,83,84,94}$ acid-base titration, ${ }^{22,54,85,86}$ and water adsorption measurements ${ }^{87,88}$ have been applied. These methods give the average defect concentration in MOFs. For example, the framework decomposition weight loss step in TGA involves the complete combustion of the organic linkers. The magnitude of this weight loss is inversely correlated with the defect density in MOF samples. ${ }^{51}$ Quantitative analysis of missing linker defects in MOFs through TGA has been widely reported..$^{\mathbf{8 1 , 8 3 , 9 4}}$

For the synthesis of defective MOFs, metal sites in MOF structures are usually capped by other species such as competitive linkers, the modulator, and/or solvents. NMR spectroscopy is a common technique to examine the relative degrees of linkers in a MOF structure and has been often used to detect the linkers and the modulator. ${ }^{\mathbf{9}, \mathbf{6 8 , 8 9}}$

Acid-base titration is another useful technique that is applicable in some specific cases. High stability of the MOF toward acids and bases is crucial for the measurement, which limits its applicability. Because different protons correspond to distinct $\mathrm{p} K_{\mathrm{a}}$ values, it is possible to identify and quantify each type of proton by titration with sodium hydroxide. ${ }^{85}$ DeStefano et al. ${ }^{54}$ reported that two new protons $\left(\mathrm{Zr}-\mathrm{OH}_{2}\right.$ and $\left.\mathrm{Zr}-\mathrm{OH}\right)$ were generated when defects were incorporated into highly stable $\mathrm{Zr}$ -
MOFs (Fig. 8a). As shown in Fig. 8b, the titration curve and its first derivative curve for defective UiO-66 are presented. Four distinct equivalence points, corresponding to $\mathrm{p} K_{\mathrm{a}}$ values of 3.34 , $4.97,6.89$, and 8.53 , were observed in the first derivative of the titration curve. They were assigned to $\mu_{3}-\mathrm{OH}$ groups in the $\mathrm{Zr}_{6}$ cluster, acetic acid, $\mathrm{Zr}-\mathrm{OH}_{2}$ and $\mathrm{Zr}-\mathrm{OH}$, respectively. The number of missing linkers per $\mathrm{Zr}_{6}$ cluster was determined as 2.6.

Defective sites in MOFs have been used to tune the hydrophilicity and wettability. ${ }^{\mathbf{1 0 5 , 1 0 6}}$ It has also been reported that
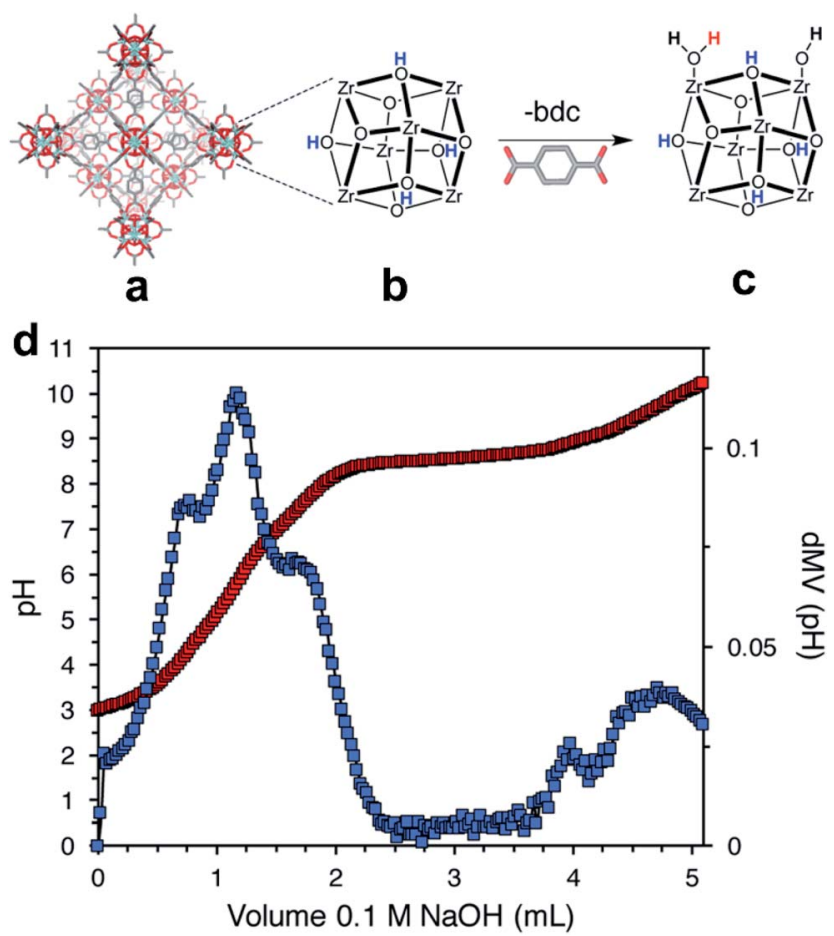

Fig. 8 (a) Structure of UiO-66, (b) an ideal 12-connected $\mathrm{Zr}_{6}$ cluster, and (c) a defective (11-connected) $\mathrm{Zr}_{6}$ cluster with $-\mathrm{OH} /-\mathrm{OH}_{2}$ capping ligands. The three types of protons in panel $\mathrm{c}$ are colored blue, red, and black for the $\mu_{3}-\mathrm{OH}$, terminal $-\mathrm{OH}_{2}$, and terminal $-\mathrm{OH}$ protons, respectively. (d) Acid-base titration curve (red) and firstderivative curve (blue). Reproduced with permission. ${ }^{54}$ Copyright 2017 , American Chemical Society. 
water adsorption measurements can be a complementary tool to analyze the defects in $\mathrm{Zr}-\mathrm{MOF}{ }^{\mathbf{8 7}, 107}$ Two simple but important parameters, Henry constant (the slope of the adsorption pressure in the low pressure range) and the saturation water uptake quantitatively represent the defect-induced hydrophilicity, which can be used to predict the catalytic properties for Lewis acid-based reactions. ${ }^{87}$

Direct spatially resolved measurement of the defect distribution is strongly desirable. It would be easier to identify local defect arrangement in microscopic detail if the defects in MOFs can be visualized. Nowadays, some emerging techniques including high-resolution transmission electron microscopy (HRTEM) ${ }^{29,48}$ confocal fluorescence microscopy (CFM), ${ }^{32,108}$ atomic force microscopy (AFM), ${ }^{109}$ fluorescence lifetime imaging (FLIM) ${ }^{110}$ and scanning electron diffraction (SED), ${ }^{111}$ are powerful for the imaging of defects in MOFs.

CFM is a unique tool for three-dimensional visualization of defects in single crystals of MOFs. ${ }^{108}$ Defects as Lewis acid sites in the MOF lattice can capture furfuryl alcohol probe molecules. Then the CFM images can locate the fluorescence signal in the oligomerization of furfuryl alcohol catalyzed by Lewis acid sites. As shown in Fig. 5f, crystal defects with uncoupled Cu(II) centers can be clearly observed by CFM analysis..$^{32}$ Wuttke et al. ${ }^{110}$ demonstrated how to use FLIM to resolve the chemical diversity of a MOF in three dimensions. Specifically, the authors revealed a correlation between fluorescence quenching and defects in UiO-67.

A HRTEM is a powerful tool to directly observe individual local structures in real space. However, MOFs are normally electron beam sensitive materials. Typically, the structures of MOFs can be significantly damaged under the electron beam in conventional HRTEM. ${ }^{112}$ Recently, novel low-dose electron microscopy techniques have been developed for the atomic resolution imaging of MOFs. ${ }^{113}$ Some bulk and local MOF structures have already been unravelled by HR-(S)TEM. ${ }^{113}$ Liu et al. ${ }^{48}$ reported the real-space observation of missing linker and missing cluster defects in UiO-66, using a combination of lowdose electron microscopy techniques and electron crystallography. Ordered missing linker and missing cluster defects were found to coexist. Similarly, Wang et al. ${ }^{29}$ investigated the detailed defect structures of UiO-66 and defective UiO-66 (denoted as UiO-66(1d)) by using HRTEM. Fig. 9 presents HRTEM images along with the simulated potential maps and the projected structural models. In Fig. 9a and b, horizontally arranged BDC linkers cannot be observed in UiO-66(1d) compared to UiO-66 (highlighted with red arrows), indicating the presence of missing-linker defects in UiO-66(1d). From the visible difference of image contrast in Fig. 9a and c, ordered missing-cluster defects in UiO-66(1d) are clearly observed.

Very recently, SED, a four-dimensional scanning transmission electron microscopy (4D-STEM) technique, has been demonstrated to be effective for the analysis of the distribution of defects or microstructures in functional MOFs. ${ }^{101}$ As shown in Fig. 10, nanoscale defect domains are directly imaged to reveal domain morphology and distribution in the MOF UiO66(Hf) over an area of $c a .1000 \mathrm{~nm}$ with a spatial resolution of $c a .5 \mathrm{~nm}$. SED provides the possibility to extend the concept of

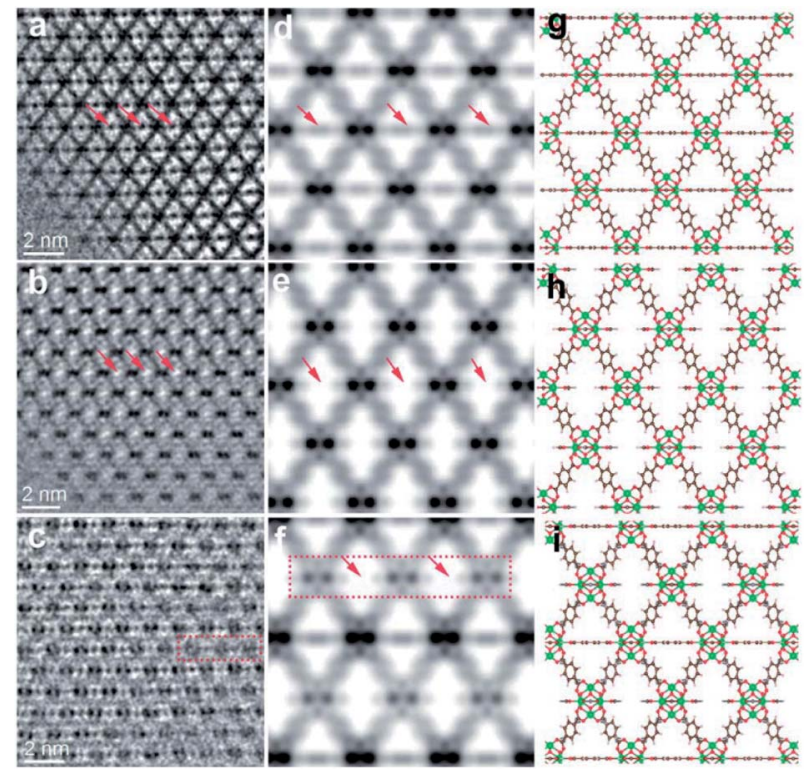

Fig. 9 HRTEM images of (a) UiO-66 and (b) missing-linker defect and (c) missing-cluster defect regions of UiO-66(1d) along the [110] zone axis; $(d-f)$ and $(g-i)$ represent relative simulated potential maps and structural models, respectively. Reproduced with permission. ${ }^{29}$ Copyright 2020, Royal Society of Chemistry.

defect engineering to microstructure engineering as a route for enhanced performance in MOFs.

Until now, various characterization techniques have been developed to investigate defect concentration and local defect arrangement in MOFs. However, it is difficult to clearly characterize defects in MOF materials by using only one characterization technique. The combination of different techniques is necessary and important to get a full picture of the structural defects in MOFs. ${ }^{29,32,57,96,114}$ The characterization of the structural defects in MOFs under real reaction or application conditions is still very challenging.

\section{Application of defects in MOFs}

It is widely recognized that structural defects in MOFs have a significant influence on their properties such as porosity, thermal and mechanical properties, electronic structures, and Lewis acidity, which may in turn affect their applications. ${ }^{47}$ Defect engineering in MOFs can bring substantial benefits for their applications. For example, additional coordinatively unsaturated sites (CUSs) can be created as active sites for adsorption and catalysis. ${ }^{5,81}$ Hierarchical micro- and mesopores can be introduced into the frameworks. These hierarchical pores provide more open accessible active sites and enhance mass diffusion as well as the loading of large molecules. In the following sections, important advances in the applications of defective MOFs are discussed.

\subsection{Catalysis}

Defective MOFs and their composites have received broad research interest in the field of heterogeneous catalysis, including oxidation, ${ }^{22,25,39,40,155,116}$ esterification, ${ }^{27} \quad \mathrm{CO}_{2}$ 

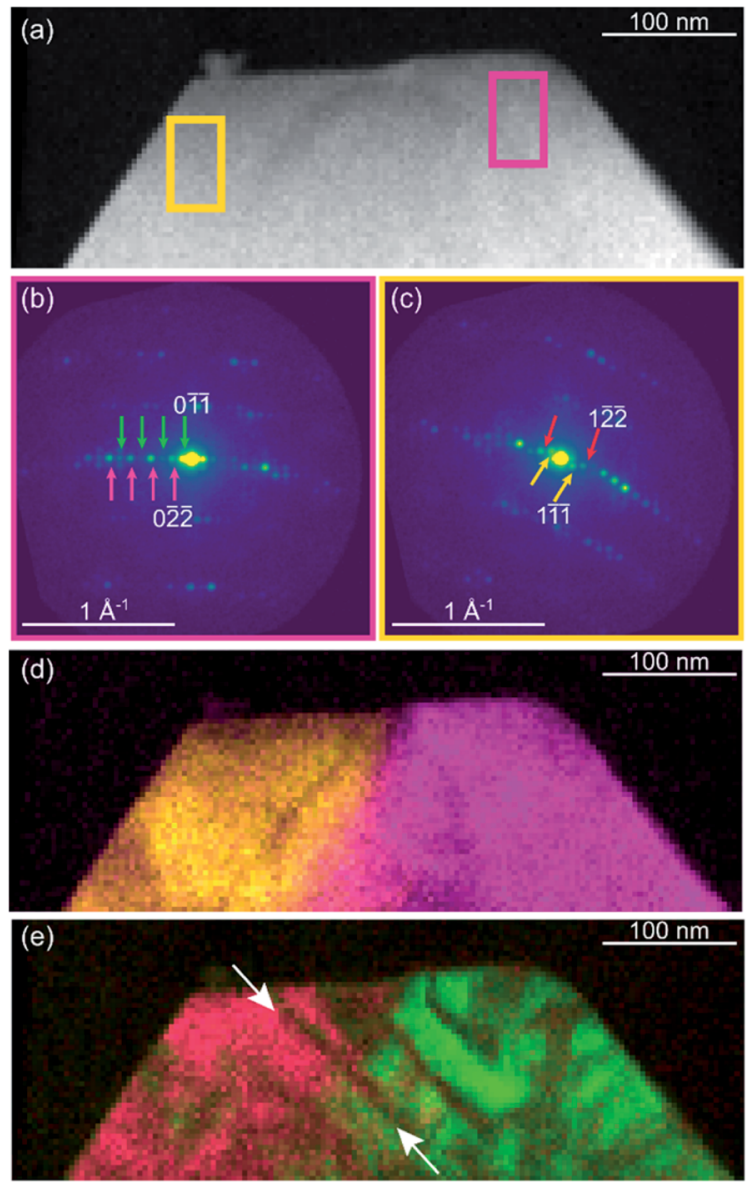

Fig. 10 (a) STEM image of the UiO-66(Hf) particle with high defect density, where integrated electron diffraction patterns (b and c) were selected in magenta and yellow. (d) Composite virtual dark-field (VDF) image formed using integration windows centered on parent reflections, magenta in (b) and yellow in (c). (e) Composite VDF image formed using integration windows centered on superlattice reflections, green in (b) and red in (c). Reproduced with permission. ${ }^{111}$ Copyright 2020, American Chemical Society.

conversion, ${ }^{36,81,117}$ and photo/electrocatalysis. ${ }^{21,23,33,118}$ The CUSs and/or the actives species immobilized in the local microenvironment of structural defects can serve as active sites in different catalytic processes.

4.1.1 Active sites based on metal centers. Introducing defects into MOFs is one of the most common methods to generate purposely CUSs around the metal ions. These CUSs can act as Lewis acid centers or create Brønsted acid centers (e.g., the terminal $-\mathrm{OH}$ groups or $-\mathrm{OH} / \mathrm{H}_{2} \mathrm{O}$ pairs on metal node defects $^{20}$ ) after exposure to protic solvents, as shown in Fig. 11a. These Lewis acids and/or Brønsted acid sites within defective MOFs play an important role in various heterogeneous catalyses. ${ }^{119}$ UiO-66 and the titanium terephthalate MOF (Ti-BDC) ${ }^{25}$ with rich defect sites were prepared by the solvent-free synthesis and acid modulator approach, respectively. Analytical data suggested that these defect sites in UiO-66 and Ti-BDC mainly consist of $\mathrm{Zr}-\mathrm{OH}$ and $\mathrm{Ti}-\mathrm{OH}$ sites, respectively. The catalytic activity of these solids was evaluated in the oxidative desulfurization (ODS) reaction. Compared to defect-free samples, the defective samples showed a better catalytic performance in the ODS reaction, which can be mainly attributed to the creation of defect sites and rich exposed active sites in these materials. These results indicate the superiority of defective MOFs in the deep ODS of oil fuel. Recently, we reported that the concentration of missing linker defects in UiO66 could be easily tuned by plasma treatment. ${ }^{81}$ The missing linker defects increase the Lewis acid sites on the surface of UiO-66, which significantly affect the catalytic performance. The defect-rich UiO-66 was confirmed to be highly active for $\mathrm{CO}_{2}$ cycloaddition with epoxides. In addition, introducing missing linker defects or CUSs has been confirmed to be an efficient route to improve the electrochemical performances of MOFs by modulating their electronic structure. ${ }^{33}$ The introduction of missing linkers provides a new alternative to develop efficient MOF-based electrocatalysts.

Recently, $\mathrm{Cu}^{+} / \mathrm{Cu}^{2+}$ dimer defects in defect-engineered MOFs of type HKUST-1 were created in a controlled fashion by oxidative decarboxylation. ${ }^{26}$ Decarboxylation leads to a reduction of the pristine $\mathrm{Cu}^{2+} / \mathrm{Cu}^{2+}$ pairs of the intact framework, thus yielding $\mathrm{Cu}^{+} / \mathrm{Cu}^{2+}$ defect pairs. Density functional theory (DFT) calculations revealed that the created defects in HKUST-1 not only yield higher binding energy adsorption sites for $\mathrm{CO}$ but also create additional space to enable the simultaneous binding of $\mathrm{CO}$ and dioxygen on $\mathrm{Cu}^{+} / \mathrm{Cu}^{2+}$ single dimers. This synergistic effect contributes to the high catalytic activity achieved in lowtemperature $\mathrm{CO}$ oxidation.

The amounts of defects in MOFs also affect their catalytic performance. Guo et $a .^{27}$ investigated the effect of defects (Lewis acid sites) in MOF supports on the catalytic activity and selectivity in the esterification of CO into dimethyl carbonate (DMC). They found that the catalytic performance is closely correlated with the number of defects in MOFs. UiO-66, MIL101, and MOF-5 with different densities of Lewis acid sites were selected as catalyst supports. The $\mathrm{Pd}(\mathrm{II}) / \mathrm{UiO}-66$ catalyst exhibited superior catalytic performance to the other catalysts (Pd(II)/MIL-101 and Pd(II)/MOF-5), with $87.92 \%$ CO conversion and $98.47 \%$ DMC selectivity (Fig. 11b). Pyridine infrared spectra were used to determine the Lewis acid strength of the three catalysts. The peak areas of pyridine adsorption at Lewis acid sites were 13.636, 5.388, and 3.398, respectively, which was consistent with the order of their catalytic performance. These results revealed that UiO-66 with more defects has more Lewis acid sites, and the catalytic activity of UiO-66 was positively correlated with the number of Lewis acid sites in MOFs. A similar finding was previously reported in UiO-type MOFs with varied amounts of missing-linker type defects based on both $\mathrm{Zr}$ and Hf nodes. ${ }^{35}$ The number of defects was found to quantitatively correlate with the catalytic activity of MOFs in an acidcatalyzed epoxide ring-opening reaction. UiO-type MOFs with a greater number of defects exhibited higher catalytic activity. However, it is important to consider the stability of defective MOF materials. For example, MOF-808 with a higher density of defect sites has shown higher activity for tert-butyl alcohol dehydration but lower stability compared to UiO-66. ${ }^{\mathbf{1 2 0}}$ Thus, an 
a<smiles>O[14CH2]O[14CH2]O</smiles>

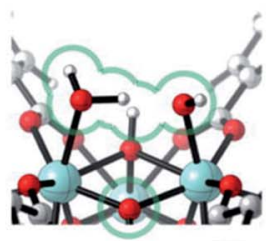

Brønsted site b

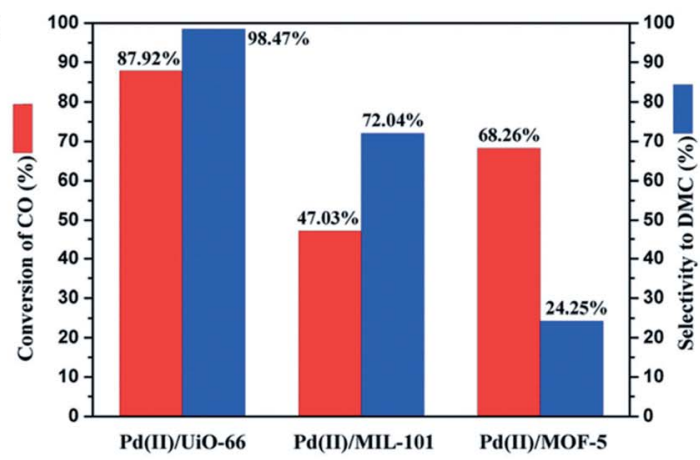

C

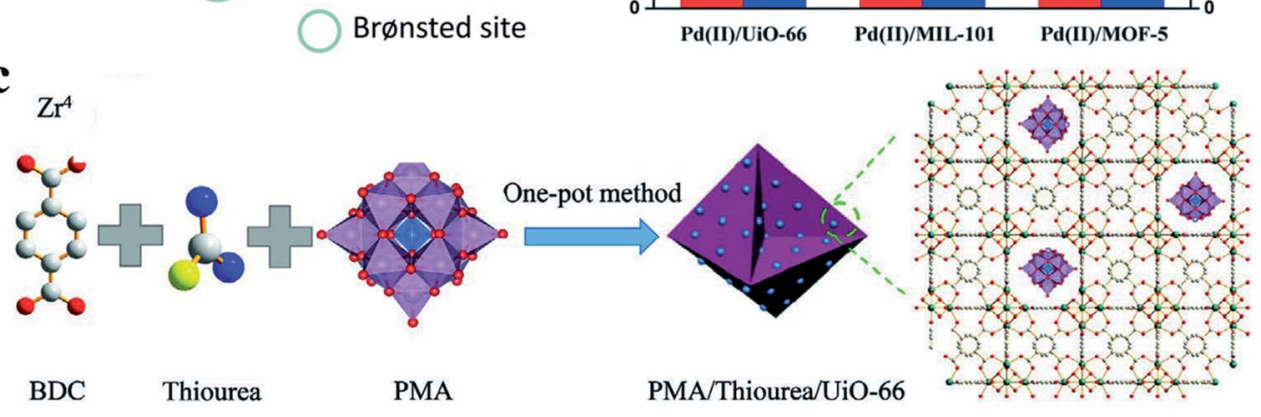

d
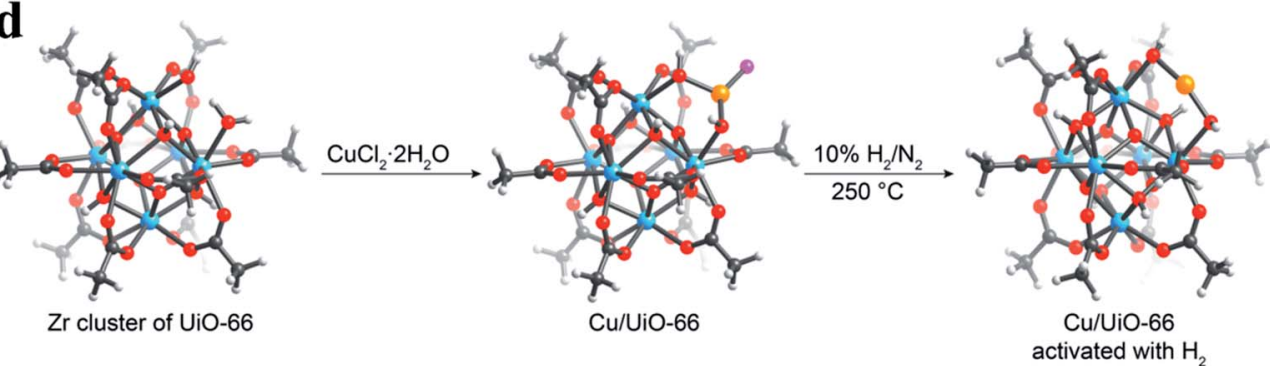

Fig. 11 (a) A calculated configuration structure of three water molecules and a coordinatively unsaturated $\mathrm{Zr}$-brick in defective UiO-66. Reproduced with permission. ${ }^{20}$ Copyright 2017, Elsevier. (b) The conversion of CO and selectivity to DMC over Pd(॥)/MOF catalysts for CO esterification to DMC. Reproduced with permission. ${ }^{27}$ Copyright 2020, Royal Society of Chemistry. (c) Preparative route for POMs/defective MOF composites. Reproduced with permission. ${ }^{37}$ Copyright 2020, Wiley-VCH. (d) The calculated structures of defective UiO-66 and a Cu/UiO-66 catalyst before and after activation with $\mathrm{H}_{2}$. Atom labeling: $\mathrm{C}$, black; $\mathrm{O}$, red; $\mathrm{Cl}$, pink; $\mathrm{Cu}$, orange; $\mathrm{Zr}$, blue. $\mathrm{H}$ : light gray. Reproduced with permission. ${ }^{39}$ Copyright 2019, American Chemical Society.

optimum defect density of MOFs should be considered to balance the catalyst activity and stability.

4.1.2 Active sites based on loading materials. Besides the catalytic activity derived from metal nodes in defective MOFs, defective MOFs have emerged as excellent hosts to support some active guest species in recent years. ${ }^{121}$ Due to their high specific surface areas and hierarchical pores, defective MOFs enable reactant molecules to access the active sites even after loaded with active guest species. ${ }^{117}$ Different kinds of guest species, such as polyoxometalates (POMs) and metal nanoparticles (NPs) have been successfully incorporated into defective MOFs. ${ }^{37-39,117,122,123}$ For example, Keggin-type POMs confined in defective UiO-66 were prepared via a one-pot method with an in situ addition of thiourea (Fig. 11c). ${ }^{37}$ When heated in an acidic solution, thiourea can hydrolyze to produce ammonia and hydrogen sulfide. These generated species in situ create missing linker defects or Lewis acid sites in the formation of UiO-66. The synergistic effect between defective UiO-66 and POM was elucidated in the ODS reaction. Additionally, a series of hierarchically porous precious NPs@MOFs (Pt@UiO-66- $\mathrm{NH}_{2}$, Pt@UiO-66, Pt@ZIF-8, and Au@ZIF-8) were successfully constructed using the thermal instability of defects around the encapsulated nanoparticles. ${ }^{38}$ After being annealed at an appropriate temperature, the generated mesopores in these MOFs can be located around the external NPs, retaining the MOF shell for catalytic selectivity. The resulting Pt@UiO-66- $\mathrm{NH}_{2}$ treated at $250{ }^{\circ} \mathrm{C}$ for $2 \mathrm{~h}$ exhibited a significantly enhanced catalytic rate and dynamic selectivity in olefin hydrogenation. Olsbye et al. ${ }^{124}$ found that Pt NPs encapsulated UiO-67 was active for $\mathrm{CO}_{2}$ hydrogenation to methanol at $170{ }^{\circ} \mathrm{C}$ and $1-8$ bar. The catalytic performance was correlated with the defect $\mathrm{Zr}$ nodes decorated on the Pt NPs surface.

In addition to the introduction of NPs into the pores of defective MOFs, the organic linkers or metal nodes can also be used to anchor some species as active sites. ${ }^{115,116,125-127}$ Yaghi et al. $^{39}$ synthesized a single-atom catalyst, $\mathrm{Cu} / \mathrm{UiO}-66$, through a covalent attachment of $\mathrm{Cu}$ atoms to the defect sites at zirconium oxide clusters (Fig. 11d). This catalyst was highly active 
and stable in $\mathrm{CO}$ oxidation even in an $\mathrm{O}_{2}$-rich atmosphere and at temperature up to $350{ }^{\circ} \mathrm{C}$. Moreover, the $\mathrm{Cu} / \mathrm{UiO}-66$ catalyst showed an excellent selectivity of about $100 \%$ in CO oxidation with $\mathrm{H}_{2}$-rich feed gases. Time-resolved operando spectroscopy revealed that the high activity of the catalyst was related to the formation of atomically dispersed positively charged $\mathrm{Cu}$ species. Chang et al. ${ }^{36}$ developed a competitive coordinationinduced defect-formation strategy to prepare hierarchically porous Cu@Al-MOF by using copper(II) tetrafluoroborate as both functional sites and etching agents. Defect-mesopores were formed within the microporous Al-MOF. Meanwhile, some of the $\mathrm{Cu}$ (II) ions were coordinated with the pyridine nitrogen within the framework (see Fig. 6a). The Cu@Al-MOF catalyst exhibited excellent catalytic activity in the $\mathrm{CO}_{2}$ cycloaddition with epoxides, which can be ascribed to the formation of hierarchical pore structures and the presence of both Lewis acid (doped copper sites) and base sites (unoccupied $\mathrm{N}$ sites). Structural defects of MOFs are attracting increasing interest for heterogeneous catalysis. ${ }^{\mathbf{1 2 8}}$

\subsection{Adsorption and separation of gases}

As a class of porous materials, MOFs are considered as promising gas adsorbents due to their excellent porosity. Until now, it has been well recognized that the defects in MOFs can alter the chemical functionalization of pores. The chemical environment of pores affects their interactions with adsorbate molecules, and consequently, the gas adsorption properties of MOFs. ${ }^{\mathbf{1 2 9 , 1 3 0}}$

As mentioned above, the defects of MOFs can provide additional active sites for the adsorption of gas molecules. Highthroughput computational screening of a large experimental MOF database identified 13 frameworks that show significantly improved methane storage capacities with linker vacancy defects. ${ }^{4}$ The simulation results of defective candidate frameworks demonstrated that the excavation of inaccessible pores via rational defect engineering can be an effective method to significantly enhance the gas adsorption performance of the existing MOFs. Kim et al. ${ }^{5}$ developed an acetic acid-fragmented linker co-assembly strategy to create mesoporous defects in microporous HKUST-1. The prepared acetic acid-fragmented HKUST-1 materials enhanced methane uptake $(13 \%$ higher storage capacity at 65 bar and 16\% higher deliverable capacity between 5 bar and 65 bar) compared to the parent HKUST-1 due to the increased surface areas and pore volumes in the defective HKUST-1. Additionally, Jiao et al. ${ }^{131}$ reported the confinement of a nanosized MOF CAU-1 into a functionalized mesoporous polymer. The confined nanosized CAU-1 possesses rich aluminium defects, which is confirmed by the ${ }^{27} \mathrm{Al}$ magic angle spinning (MAS) NMR characterization. The hybrid composite exhibits significantly enhanced $\mathrm{CO}_{2}$ adsorption capacity at low pressure due to the favorable diffusion kinetics and the presence of metal defects as active binding sites.

In addition, the functionalization of MOFs with different groups has been demonstrated to increase the affinity for some specific gas molecules. Recently, amino functionalities were introduced into defective UiO-66 by post-synthetic ligand exchange. The process involves the substitution of the formic a

b
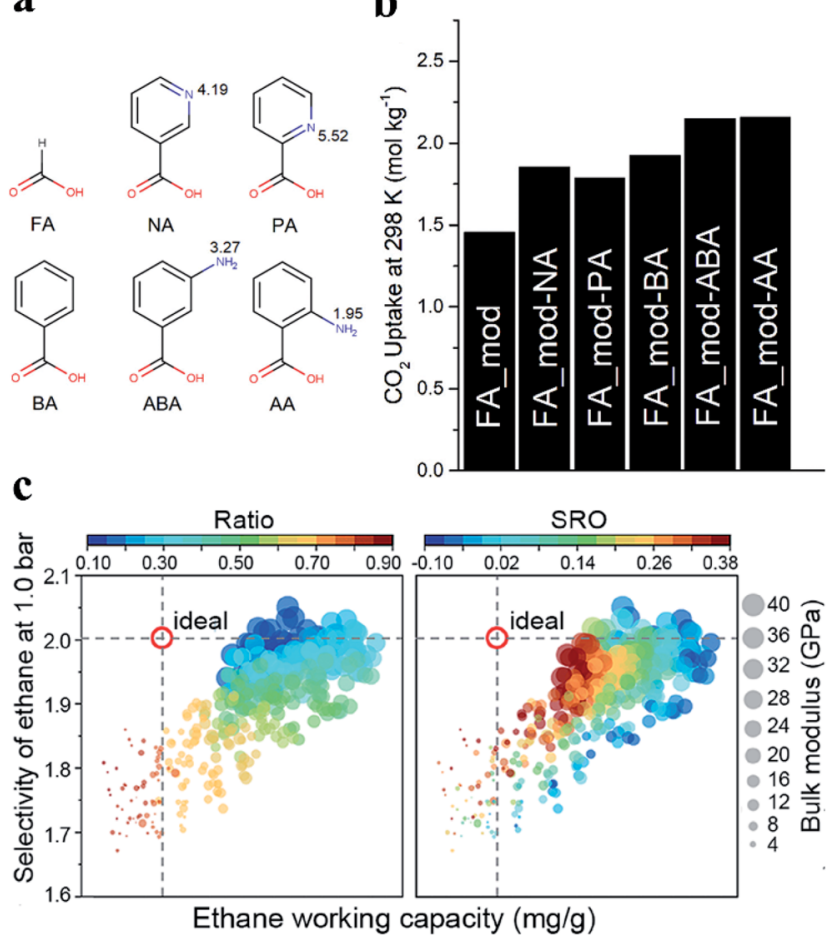

Fig. 12 (a) Chemical structures of the carboxylic acids: formic acid $(F A)$, picolinic acid (PA), nicotinic acid (NA), 3-aminobenzoic acid (ABA), anthranilic acid (AA) and benzoic acid (BA). (b) $\mathrm{CO}_{2}$ uptake values in mol kg $\mathrm{kg}^{-1}$ at $2{ }^{\circ} \mathrm{C}$ and 1 bar. Reproduced with permission. ${ }^{6} \mathrm{Copy}-$ right 2019, Royal Society of Chemistry. (c) Scatter plots for the structures of UiO-66-Ds referring to ethane work capacity and selectivity at 1.0 bar. The size of the data points indicates the bulk modulus. A range of missing-linker ratios and short-range orders (SRO) are for quantifying the concentration and distribution of defects. Reproduced with permission. ${ }^{132}$ Copyright 2020, American Chemical Society.

acid modulator grafted on CUSs with various nitrogencontaining monocarboxylates (Fig. 12a). ${ }^{6}$ The parent UiO-66 (denoted as FA_mod) was prepared with FA as a modulator. The products obtained by the exchange of the FA species in FA_mod with 3-aminobenzoic acid or anthranilic acid were denoted as FA_mod-ABA and FA_mod-AA, respectively. As shown in Fig. 12b, FA_mod-ABA and FA_mod-AA significantly enhanced the $\mathrm{CO}_{2}$ uptake $\left(2.14 \mathrm{~mol} \mathrm{~kg}^{-1}\right.$ ) by almost $50 \%$ compared to that of the parent FA_mod $\left(1.44 \mathrm{~mol} \mathrm{~kg}^{-1}\right)$ at $1 \mathrm{bar}$ and $25{ }^{\circ} \mathrm{C}$. Erkartal et al. ${ }^{9}$ introduced boronic acid moieties as functional defects into UiO-66 using a mixed-linker method. The accessible boronic acid moieties on the pore surfaces significantly improved the uptake of hydrogen. A significant increment in $\mathrm{CO}_{2}$ selectivity using the resulting MOFs over $\mathrm{N}_{2}$ and $\mathrm{CH}_{4}$ was observed, and this can be attributed to the quadrupolar interaction between the active surfaces of UiO-66 and $\mathrm{CO}_{2}$ molecules.

Structural defects of MOFs also play a vital role in gas separation. Currently, ethane-ethylene separation is an emerging topic for gas separation. Recently, $\mathrm{Wu}$ et al. ${ }^{\mathbf{1 3 2}}$ reported that machine learning could provide data-driven insight into how the defects control the performance of defective UiO- 
66 (UiO-66-Ds) in ethane-ethylene separation. They created a modeling library containing 425 UiO-66-Ds with a comprehensive population (in terms of concentration and distribution) of missing-linker defects. Fig. 12c gives a general view of ethane-ethylene separation performance using UiO-66-Ds. The structures in the top right of each panel exhibited a more desirable working capacity and selectivity, as well as comparable mechanical stability with respect to the ideal UiO-66. These privileged structures have relatively low concentrations of defects (missing-linker ratios of $0.2-0.3$ ), but the defects are prone to be distributed randomly $(\mathrm{SRO}=0.02)$ at $1.0 \mathrm{bar}$. The authors found that controlling the concentration of the defects was more significant in tuning the overall properties compared to the distribution of the defects.

\subsection{Decontamination}

The defect-induced active sites in MOFs, together with their high porosity make these materials promising in the field of decontamination. The decontamination of chemical warfare agents (CWAs) $)^{\mathbf{2 4 1 3 3 - 1 3 5}}$ and the adsorption of pollutants (e.g., organoarsenic compounds ${ }^{11,136}$ and dyes ${ }^{137,138}$ and gas pollutants $\mathbf{6}^{6,731,83,97,139,140}$ ) have been widely investigated with the use of defective MOFs. Recently, zirconium-based MOFs (Zr-MOFs) with open metal sites and excellent stability have not only shown efficient uptake of CWAs but also enhanced the chemical detoxification of organophosphorus-based CWAs in a hydrolysis reaction. ${ }^{\mathbf{2 4 , 1 3 4 , 1 4 1}}$ DFT calculations indicate that the mechanism of the hydrolysis reaction involves the binding of nerve agents on Zr-MOF secondary building units (SBUs) followed by the hydrolysis of the phosphoester bond (Fig. 13a). ${ }^{142,143}$ It has been found that increasing accessibility to $\mathrm{Zr}_{6}$ active sites enhances the rates of hydrolysis for nerve agents and their simulants. The introduction of defects into Zr-MOF generates hierarchically porous structures with additional active sites for the hydrolysis. The hierarchical pores can improve accessibility to the $\mathrm{Zr}_{6}$ active sites. For example, Peterson et al. ${ }^{\mathbf{1 3 4}}$ prepared a series of UiO-66$\mathrm{NH}_{2}$ materials with different defect concentrations and pore sizes by varying the amount of modulator and the synthetic temperature. When using these materials as catalysts for the
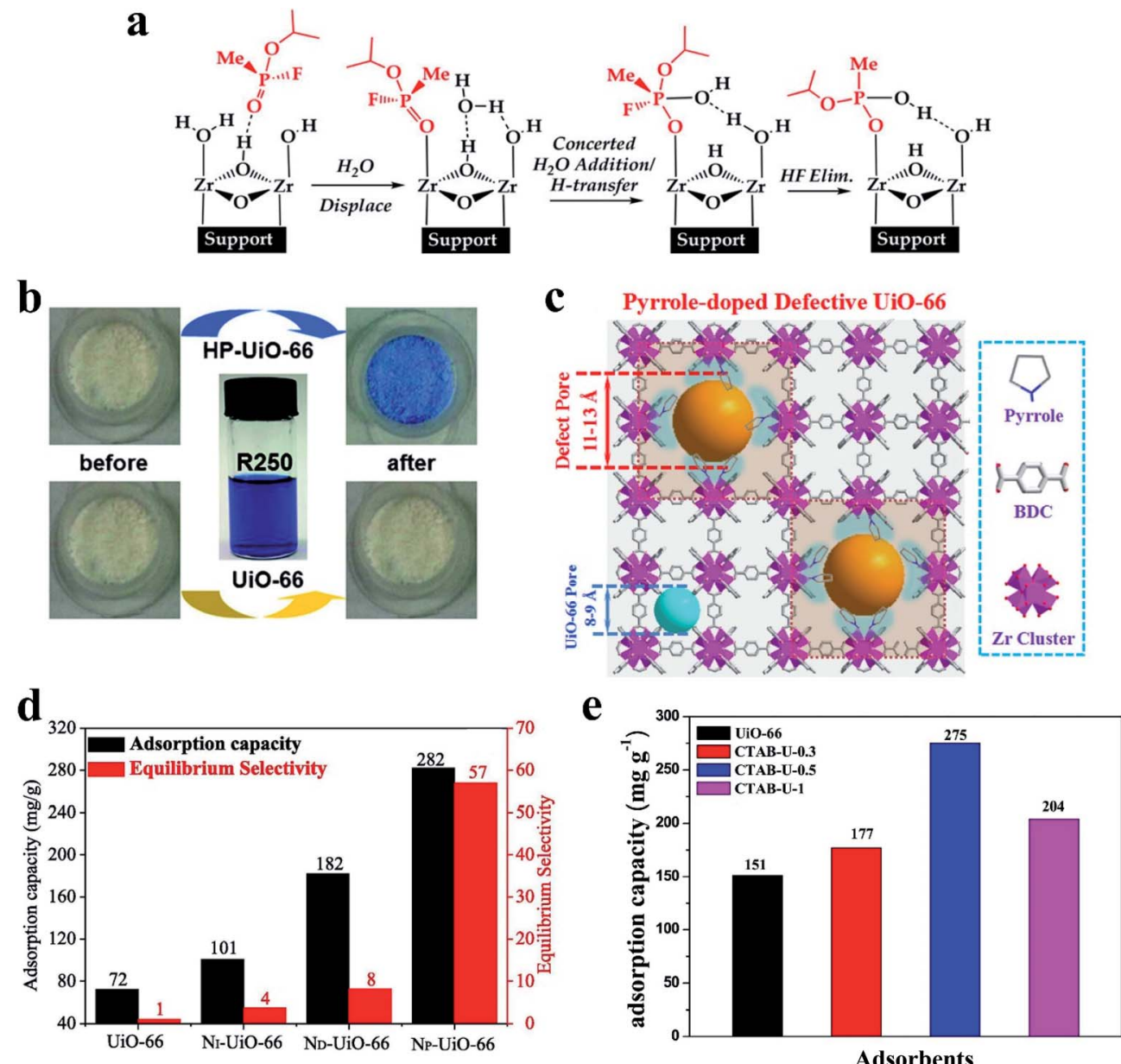

Adsorbents

Fig. 13 (a) A mechanistic scheme for the hydrolysis of sarin on uncapped faces of Zr-MOFs. Reproduced with permission. ${ }^{143}$ Copyright 2018 , American Chemical Society. (b) The color change of UiO-66 and HP-UiO-66 before and after dye adsorption. Reproduced with permission. ${ }^{53}$ Copyright 2017, Wiley-VCH. (c) Defect pore creation in UiO-66 via pyrrole in situ doping. (d) RhB adsorption and corresponding equilibrium selectivity (RhB/ST) on UiO-66 and $\mathrm{N}_{\mathrm{X}}-\mathrm{UiO}-66$. Reproduced with permission. ${ }^{146}$ Copyright 2019 , Elsevier. (e) The toluene sorption capacity for different UiO-66 samples. Reproduced with permission. ${ }^{83}$ Copyright 2018, Elsevier. 
hydrolysis of dimethyl 4-nitrophenylphosphate (DMNP), soman (GD), and $o$-ethyl $S$-[2-diisopropylaminoethyl]methylphosphonothioate (VX), the half-life $\left(t_{1 / 2}\right.$, time needed to hydrolyze $50 \%$ of the substrate) of low-defect UiO- $66-\mathrm{NH}_{2}$ for DMNP, GD, and VX is 495, 385, and $770 \mathrm{~min}$, respectively. However, significant improvement (by more than 20 times) of the half-life on high-defect UiO-66- $\mathrm{NH}_{2}$ was observed $\left(t_{1 / 2}=20\right.$, 19, 31 min for DMNP, GD, and VX, respectively). Momeni et al. ${ }^{143}$ predicted that the reactivity of the $\mathrm{Zr}_{12}$-bi-defective system in the hydrolysis of the sarin nerve agent is higher than that using other coordinatively unsaturated $\mathrm{Zr}_{6}$-MOFs including MOF-808, NU-1000, and defective UiO-66 from both periodic and extended cluster calculations.

Clearly, defect-formation strategies can create hierarchical pores and defect sites in MOFs. Missing linkers and/or missing clusters generate more open frameworks and thus enhance mass transfer diffusion and the adsorption. Shi et al. ${ }^{94}$ calculated the migration energy of a uranyl ion in both perfect and defective UiO-66 using classical molecular dynamics simulations. The free energy of the uranyl ion diffusion in the ideal UiO-66 framework was about $31 \mathrm{kcal} \mathrm{mol}^{-1}$. When one aromatic linker was missing to broaden the window size, the energy value dramatically decreased to $17.4 \mathrm{kcal} \mathrm{mol}^{-1}$. Besides, recent studies have confirmed that defect-formation hierarchical pores provide an efficient route for the adsorption of molecules with a large size (like organic dyes). For example, defect-induced hierarchically porous UiO-66 (HP-UiO-66) shows its advantages for dye uptake with coomassie brilliant blue R250 (R250, ca. $2.7 \times 1.7 \times 0.9 \mathrm{~nm}$ in size). ${ }^{53}$ Upon soaking HP-UiO66 in a solution of R250, the color of the dye solution faded while the white powder turned blue during dye uptake. In stark contrast, the pristine UiO-66 (window size, $c a .0 .6 \mathrm{~nm}$ ) cannot uptake such a large molecule, and its color remained unchanged (Fig. 13b). Similarly, due to the size-exclusion effect constructed from the defects in the framework, high selectivity for safranine T (ST) over crystal violet was observed in defective UiO-66. ${ }^{144}$ When using defective MOFs for the adsorption of organic dyes and organoarsenic compounds, the pore size, the number of defects, and electronegativity of the defective MOFs are of great importance in determining the adsorption performance. ${ }^{137,144,145}$ Furthermore, surface alkalinity of the defective UiO-66 has a notable effect on its selective adsorption for cationic dyes (rhodamine B (RhB) and ST) with similar sizes. ${ }^{146}$ Alkaline N-compound (pyrrole, dopamine, and 2-methylimidazole) coordination was proved to simultaneously modulate pore sizes and intensify surface alkalinity of the original UiO-66 (Fig. 13c), significantly decreasing the adsorption interaction towards basic dyes. As shown in Fig. 13d, pyrrole coordinated UiO-66 ( $\mathrm{N}_{\mathrm{P}}$-UiO-66) exhibited approximately three times enhanced adsorption capacity for $\mathrm{RhB}\left(282 \mathrm{mg} \mathrm{g}^{-1}\right)$ and 56 times higher selectivity for the equimolar RhB/ST binary system than that of the parent UiO-66.

Defective MOFs have also been used as adsorbents for the adsorption of gaseous pollutants such as $\mathrm{H}_{2} \mathrm{~S}, \mathrm{SO}_{2}$, and volatile

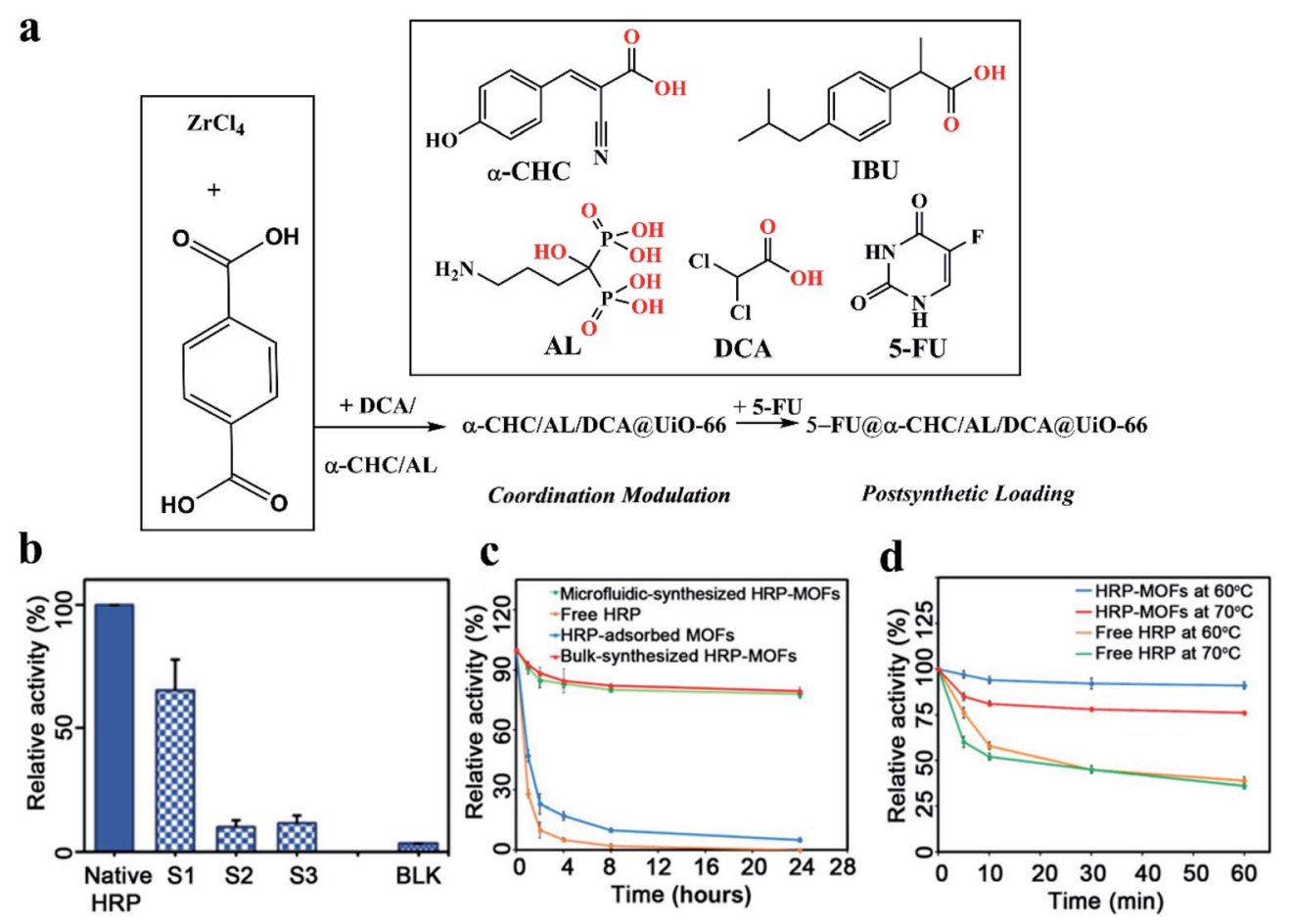

Fig. 14 (a) Synthesis of drug modulated MOFs and their post-synthetic drug loading. The chemical structures (coordinating groups in red) and abbreviations of the drugs are shown in the inset. Adapted with permission. ${ }^{52}$ Copyright 2020, Wiley-VCH. (b) The relative enzyme activity of free HRP, microfluidic flow-synthesized HRP-MOF composites, and bulk solution-synthesized HRP-MOF composites. (c) The relative enzyme activity. (d) The thermal stability of free native HRP and HRP-MOF composites at $60^{\circ} \mathrm{C}$ and $70{ }^{\circ} \mathrm{C}$, respectively. Reproduced with permission. ${ }^{30}$ Copyright 2020, American Association for the Advancement of Science. 
organic compounds (e.g., benzene)., ${ }^{7,8,31,41,97,140}$ The deliberate creation of defects in MOFs significantly enhances the gas adsorption capacity (e.g., $\mathrm{SO}_{2}$ ) due to the enhanced pore accessibility and the specific interactions between sulfur dioxide molecules and defect sites. ${ }^{8}$ The dependence of $\mathrm{H}_{2} \mathrm{~S}$ absorption on the density of the defects in HKUST-1 and MIL101 has been systematically investigated. ${ }^{7,31}$ Recently, defective UiO-66 prepared using a simple solvothermal method with structure-directing agents (e.g., P123, ${ }^{97}$ polyvinylpyrrolidone,${ }^{139}$ and cetyltrimethylammonium bromide $\left.(\mathrm{CTAB})^{83}\right)$ showed excellent toluene adsorption performance. As shown in Fig. 13e, defective UiO-66 (CTAB-U-0.5) exhibited much higher adsorption of toluene compared to pristine UiO-66 $\left(275 \mathrm{mg} \mathrm{g}^{-1} \mathrm{vs}\right.$. $151 \mathrm{mg} \mathrm{g}^{-1}$ ). Defect sites present in UiO-66 serve as the main active adsorption sites for the effective adsorption of toluene vapor and enhance the interaction between the defective UiO-66 and toluene molecules. The micro-mesoporous structure of UiO-66 has a positive effect on the toluene molecule capture. ${ }^{97}$

\subsection{Bio-applications}

MOFs have been proposed as attractive carriers of drugs and biomolecules due to their low toxicity, good clearance, and high loadings. The formation of defects in MOFs further opens a broad range of opportunities to design novel drug or biomolecule loaded MOFs. The multimodal distribution of pore size in defective MOFs can improve the access of substrates to encapsulated active molecules. ${ }^{30,79,147}$ Teplensky et al. ${ }^{148}$ developed a temperature treatment process to delay the release of a model drug compound calcein as well as the anticancer therapeutic alpha-cyano-4-hydroxycinnamic acid ( $\alpha$-CHC), which can be attributed to the partial collapse of pores in the MOFs as a result of the increased temperature. The anticancer drug showed improved efficacy when loaded into the temperature-treated defective MOFs, although the authors did not emphasize the importance of defects in MOFs. This work shows that the defect engineering of MOFs has great potential to offer an emerging solution for the long-term controlled release of drugs. Recently, Forgan et $a l^{52}$ incorporated up to three drugs into the UiO-66 by defect-loading in a one-pot synthesis, in which multiple drugs possessing carboxylate and phosphonate-containing moieties were used as both drug molecules and modulators (Fig. 14a). These drugs were distributed throughout the MOF at defect sites by coordination to the metal clusters rather than being pore-loaded, leading to retention of crystallinity and porosity. The retained porosity was used to post-synthetically load the fourth drug 5-FU into the MOFs (Fig. 14a) to yield NPs loaded with cocktails of drugs that show enhancements in selective anticancer cytotoxicity against MCF-7 breast cancer cells in vitro. Multivariate modulation is a significant advancement in the application of MOFs in biomedicine and can also be adopted in other areas of MOF chemistry.

Moreover, as mentioned above in Fig. 5a, the microfluidic flow synthesis method ${ }^{\mathbf{3 0}}$ was developed to produce highly active enzyme-MOF composites with a multimodal distribution of pore size induced by generated defects. The microfluidic flow- synthesized horseradish peroxidase-MOF (HRP-MOF) composites showed much higher (approximately one order of magnitude) biological activity than those prepared using a conventional bulk solution synthesis approach (see S1 vs. BLK, Fig. 14b). The increased activity can be ascribed to the formation of defects in MOFs, which enhances the diffusion of substrates. Meanwhile, because of the MOF protection of the enzymes, the HRP-MOF composites exhibited superior stabilities at high temperatures and were more resistant to protease digestion compared to their natural counterparts (Fig. 14c and d). Yang et al. ${ }^{149}$ constructed a multifunctional MOF structure (MIL-53(Fe)) with near-infrared dye (cypate) based on the interaction between $\mathrm{Fe}^{3+}$ and the carboxyl group of cypate molecules. This MOF material can achieve multimodal imaging and guided phototherapy. Moreover, the tumors are entirely eliminated without noticeable side effects in the precise cancer phototherapy, indicating the high efficacy and safety of this multifunctional MOF platform.

\subsection{Smart applications}

4.5.1 Tuning the mechanical response. It is necessary to consider the mechanical properties or stability issue of MOFs when exposed to significant mechanical stress during industrial extrusion processes. Hence, understanding how defective MOFs respond to stress is essential to their successful commercialization. Zheng et al. ${ }^{18}$ investigated the deformation mechanisms of defective MOF-5 using molecular dynamics simulation. The presence of defects in MOF-5 reduces the yield strength via inducing the nucleation of dislocations. Experimental evidence for the impact of defects on the compression of UiO-66 was reported by Dissegna et al. ${ }^{19}$ The bulk modulus decreases with the increase of the missing linker defect density from $3 \%$ to $26 \%$. However, at higher defect densities (28\% missing linkers), the bulk modulus of UiO-66 appeared to increase (Fig. 15a). These results help us further understand how the presence of defects affects the mechanical properties of MOFs.

4.5.2 Tuning the electronic band structure. Recent studies have reported that the defects in MOF materials can alter their electronic band structure. ${ }^{\mathbf{1 3 - 1 5 , 2 3}}$ For example, a defect engineered Zr-MOF UiO-66 was obtained by introducing a range of amino-functionalized benzoic acids as defect compensating species, featuring a band gap in the $3.3-4.1 \mathrm{eV}$ range. ${ }^{13}$ In our recent work, we used plasma etching for the rapid generation of missing linker defects in UiO-66. ${ }^{81}$ The concentration of the defects can be tuned by changing the etching time, while the band gap of the defective UiO-66 decreased with an increase in the number of defects (Fig. 15b). The theoretical calculations further confirm the influence of defects on the electronic structures of MOFs. De Vos et al. ${ }^{15}$ investigated the effect of missing linker defects on the electronic structures of UiO-66 by using periodic calculations. They found that the coordination of the constituent nodes, especially the nodes with the strongest local distortions alter the electronic structure of UiO-66. It is expected that the defects provide an alternative pathway to modulate the electronic structure of MOFs and thus their photocatalytic and electrocatalytic activities. ${ }^{13,23}$ Recently, Jiang 

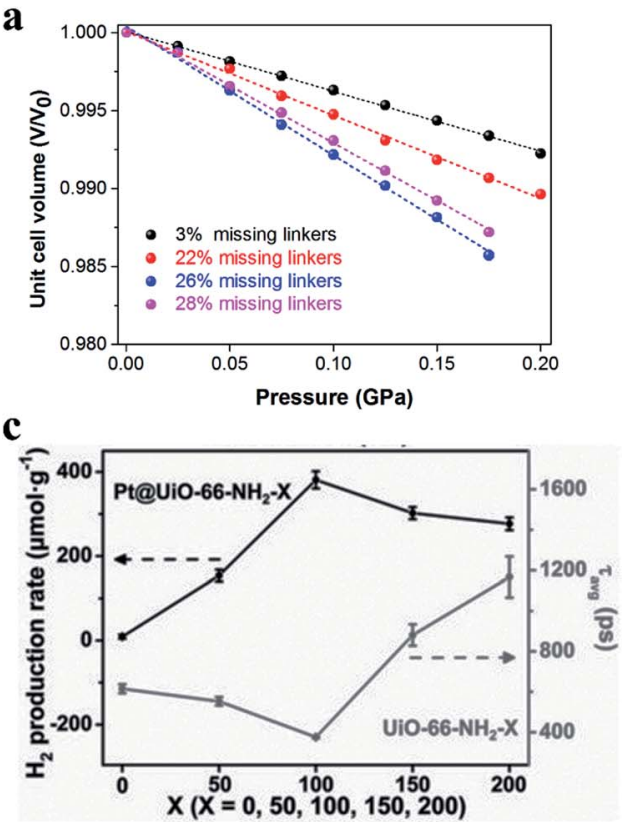
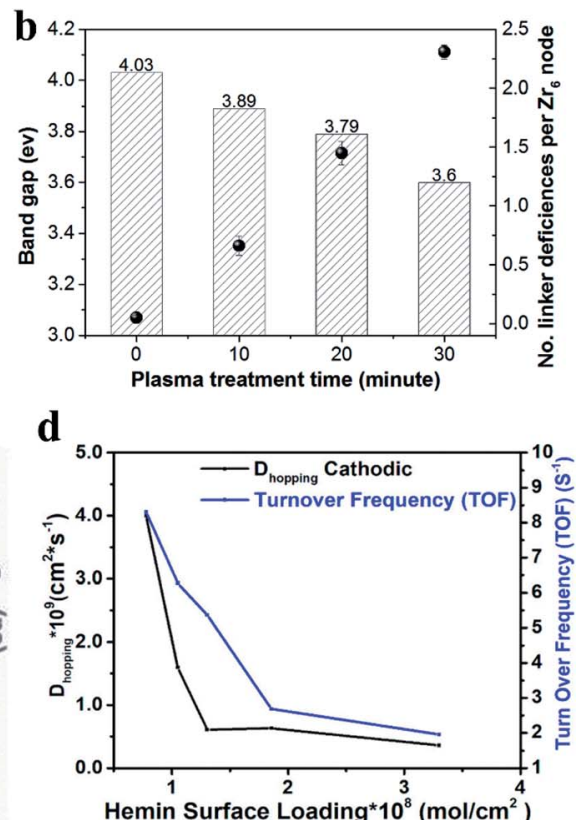

Fig. 15 (a) Relative volumes as a function of pressure for UiO-66 with different defect densities. Steeper slopes correspond to lower bulk modulus values. Adapted with permission. ${ }^{19}$ Copyright 2018, American Chemical Society. (b) The calculated band gaps and the number of linker deficiencies per $\mathrm{Zr}_{6}$ node vs. the plasma treatment time. Adapted with permission. ${ }^{81}$ Copyright 2020, Elsevier. (c) Comparison of photocatalytic $\mathrm{H}_{2}$ production rates and average relaxation lifetimes. Reproduced with permission. ${ }^{23}$ Copyright 2019, Wiley-VCH. (d) A plot of ORR turnover

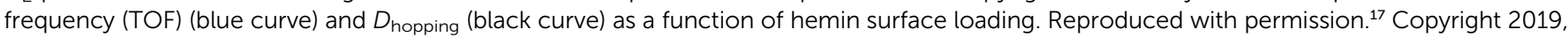
American Chemical Society.

et al. ${ }^{23}$ demonstrated that the formation of structural defects with optimized contents is vital in determining the photocatalytic activities of Pt@UiO-66- $\mathrm{NH}_{2}$. As shown in Fig. 15c, the photocatalytic $\mathrm{H}_{2}$ production rate presents a volcano-type trend with incremental contents of structural defects in UiO-66- $\mathrm{NH}_{2}$. The volcano-type trend of $\mathrm{H}_{2}$ production rates shows an exactly reverse relation to the average relaxation lifetime. The faster the relaxation, the higher the efficiency. Recently, the calculations and experimental studies have demonstrated that the electrocatalytic performance of MOFs can be controlled by regulating the electronic structure of MOFs via defect engineering. Xue et $a l .{ }^{33}$ and $\mathrm{Yu}$ et $a l .{ }^{44}$ demonstrated that the activity of CoBDC$\mathrm{NF}$ in an oxygen evolution reaction (OER) could be facilitated by tuning the electronic structure of CoBDC-NF through the creation of missing linker defects. After introducing missing linkers (Fc), CoBDC-Fc-NF shows significantly enhanced OER activity with an ultralow overpotential of $178 \mathrm{mV}$ to achieve $10 \mathrm{~mA}$ $\mathrm{cm}^{-2}$, which is 74 and $57 \mathrm{mV}$ lower than that of CoBDC-NF and commercial $\mathrm{RuO}_{2}$, respectively.

4.5.3 Tuning the conductivity. Despite the fact that MOFs have shown significant potential in a wide range of applications, the majority of MOF materials are electrical insulators due to the inherent chemical bonding in MOFs, i.e., the coordination bond chemistry. ${ }^{47}$ However, it is possible to tune the charge transport in MOFs by judiciously designing frameworks, including intentionally incorporating guest molecules/ions, functionalized groups, and defects. ${ }^{16,17}$ Montoro et al. ${ }^{16}$ investigated the effect of defects on the hydroxide-ion conductivity of MOF materials $\left[\mathrm{Ni}_{8}(\mathrm{OH})_{4}\left(\mathrm{H}_{2} \mathrm{O}\right)_{2}\left(\mathrm{BDP}_{-} \mathrm{X}\right)_{6}\right]\left(\mathrm{H}_{2} \mathrm{BDP} \_\mathrm{X}=1,4-\right.$ bis(pyrazol-4-yl)benzene-4-X, $\mathrm{X}=\mathrm{H}, \mathrm{OH}, \mathrm{NH}_{2}$ ) as well as their modified materials. After a post-synthetic treatment with potassium hydroxide in ethanol, missing linker defects were introduced into the frameworks due to partial linker dissolution, enhancing the overall porosity. The enhanced porosity in these materials has a positive impact on the mobility of charge carriers. Another considered favorable factor was the enhanced basicity and hydrophilicity of these materials due to the deprotonation of coordinated water molecules on metal nodes and the incorporation of an extra-framework of potassium ions. As a consequence of the higher porosity, increased basicity and hydrophilicity, a significant enhancement of conductivity up to $1.16 \times 10^{-2} \mathrm{~S} \mathrm{~cm}^{-1}$ (activation energy $E_{\mathrm{a}}=0.20 \mathrm{eV}$ ) was achieved at a relative humidity of $100 \%$. This conductivity is three to four orders of magnitude higher than that of the pristine materials. Hod et al. ${ }^{17}$ modulated the spatial distribution of a post synthetically installed molecular catalyst (hemin) by systematic control of MOF defect site density. As a result, variances in counterion diffusion rates toward bulk or surface installed electroactive hemins changed the charge transport rates by an order of magnitude. Moreover, they found a direct correlation between the tuned redox-based conductivity and the resulting oxygen reduction reaction activity (Fig. 15d).

\section{Conclusions and future perspectives}

After summarizing the recent advances in the synthesis, characterization and application of defective MOFs, the following conclusions can be made. 
(1) The de novo synthesis and the post-synthetic treatment are commonly used as effective approaches for the generation of defects in MOFs. The modulator approach, mixed-linker approach and some novel methods (like electrosynthesis) can effectively promote the generation of defects during the de novo synthesis of MOFs. Post-synthetic treatment involves a heterogeneous treatment of the parent MOFs with modified linkers, etching agents, or other activation procedures.

(2) Defect engineering of MOFs provides a powerful tool to create and tune the physical-chemical properties of MOFs, such as the electronic band structure, conductivity, and mechanical stability.

(3) The conventional spectroscopic and microscopic characterization methods with some emerging techniques have been demonstrated to be powerful for the analysis of the defects (e.g., the defect concentration and spatial distribution) in MOFs. These cutting-edge characterization techniques will play a more critical role in getting new insights into defect engineering with the rapid development of defective MOFs.

(4) Defective MOFs and their composites have shown a wide range of applications not limited to adsorption, separation, catalysis, decontamination and biological medicine. Defective MOFs is an excellent connection between homogeneous catalysts and heterogeneous ones. We would expect increasing applications of defective MOFs and their composites.

(5) Structural defects create additional coordinatively unsaturated metal sites (CUMs) and hierarchically micro- and mesopores in the frameworks. The additional CUMs serve as important sites for adsorption, catalysis, and decontamination as well as anchoring nanoparticles to prepare effective NPs/ MOF catalysts. Defective MOFs can also be excellent supports for the synthesis of single atom catalysts and photo/ electrocatalysts. These hierarchical pores provide more open, accessible active sites and enhance mass diffusion as well as the loading of large molecule objects in MOFs.

Nowadays, defect engineering of MOFs is an emerging research topic with growing interest. Significant efforts have been devoted to investigating the roles of defects of MOFs in different applications. However, the fundamental understanding of defects in MOFs and their composite materials is still in its infancy. Significant knowledge gaps in MOF defects still exist. More fundamental studies on the defects of MOFs are critical to bring the gap and develop more in-depth insights into the emerging area. The following studies are expected to achieve further advance in defective MOFs:

(1) Defect engineering has been recognized as a useful tool to tune the properties of MOFs. Both theoretical and experimental studies have demonstrated that the properties of MOFs are determined by both the concentration and spatial arrangement of missing linker defects in MOFs. Nowadays, tuning the concentration of defects in MOFs can be achieved experimentally using appropriate synthetic techniques. However, controlling the location and spatial distribution of defects in MOFs remains a challenge. More studies are needed.

(2) New synthetic methods that produce controllable structural defects in MOFs should be developed. Irradiation, local spot treatments (like laser, ${ }^{\mathbf{6 8}}$ site limited plasma treatment, ${ }^{\mathbf{1 5 0}}$ heat and chemical treatments), photochemistry, selfassembly, ${ }^{151}$ and three-dimensional printing ${ }^{152}$ may provide opportunities for effective defect formation and spatial arrangement in MOFs. The synthesis of MOFs with a single missing linker or single missing cluster would generate further research interest in defect chemistry with more unique applications.

(3) The majority of studies on the defect engineering of MOFs are performed on a small scale in the laboratory. Considering the potential for large-scale applications, although an acid/base co-modulation strategy has been developed for large-scale synthesis of monodisperse UiO-66 octahedral crystals with tunable sizes and missing linker defects, ${ }^{84}$ more efforts are needed to develop and explore synthetic routes for largescale production of defective MOFs with controllable and reproducible defects.

(4) Detection and characterization of defects are crucial for MOF defect studies. Conventional characterization methods are not sufficient to fully understand the nature of defects and the relationship between defects and MOF properties. For example, it is still unclear whether both the missing-linker and missingcluster defects can be exclusively distinguished from each other as a result of their complexity of coexistence. Novel and powerful characterization methods are desired to get new insights into the defect structure-property relation. Several currently nonstandard characterization methods such as neutron diffraction, SED, and anomalous diffraction are expected to generate valuable information in the future. ${ }^{47,111}$ It is beneficial to further understand the nature of defects in MOFs with the development of characterization techniques. At the same time, characterization methods are critical to get a further in-depth understanding of the MOF crystallization mechanism and the nature of compensating species at defective sites under complicated in situ synthesis conditions. ${ }^{\mathbf{4 3 , 1 5 3}}$

(5) Computational chemistry also plays an important role in getting new insights into defect-property relations. Some successful cases have been previously reported in the literature,$^{47}$ such as the effect of missing linker defects on both the gas sorption properties and mechanical stability of MOF materials. ${ }^{10}$ Theoretical investigation can predict new structures and properties of defective MOFs through high-throughput screening with higher efficiency but less resource consumption compared to conventional experiments. ${ }^{132}$ In addition, theoretical calculations play an increasingly important role in profoundly understanding the reaction mechanism of defective MOFs in adsorption, catalysis and others. More efforts should be devoted to investigating new and stable defective structures of MOFs. In the future, with the development of machine learning, data-driven computational chemistry has great potential for the effective design of stable defective MOF materials. However, it is also noted that a uniform distribution of defects has always been considered in the computational modeling of MOFs due to the limitation of periodic calculations, which is not the case in the experimental characterization of defects in MOFs. Thus, developing more comprehensive and accurate theoretical models considering defect concentration 
and chemistry is critical to get new insights into the defect engineering of MOF materials.

(6) Until now, zirconium-based MOFs, especially UiO-66 have been the major focus in defect engineering of MOFs. ${ }^{41,43}$ UiO-66 has been considered as a standard platform for defect engineering studies due to its high tolerance to the structural defects and excellent synthetic tunability. A new MOF platform should be developed for defect engineering studies, which will enable us to understand if the defect-property relations are consistent among different systems, or if it is necessary to look at each system independently.

(7) The incorporation of active guest species with large size into microporous MOFs is expected to be achieved by missinglinker defect formation and following defect repair in the near future. ${ }^{57,58}$ This preparation method provides an opportunity to avoid the leaching of active guest species from MOF pores. Moreover, structural defects in MOFs provide unique local environments for the immobilization of guest species and even for the modification of the electronic state of metal NPs. ${ }^{154}$ It is expected to fully utilize MOF defects from different aspects in future.

(8) The publications on the use of MOFs in thermal catalytic reactions have increased significantly since 2011. ${ }^{155-157}$ Many MOFs, even the most carefully synthesized MOFs, are known to contain structural defects even under ambient conditions. It is possible that some kinds of 'intermediate' defects could be generated at elevated temperatures. Thus, it is critical to understand the behaviors of MOFs under real reaction conditions using operando and in situ tools, which is the key for the future development of highly active and stable MOF-based catalysts.

In conclusion, defects play important roles in the enhancement of performances of MOFs. Defective MOFs will have more future applications with the new findings of properties induced by structural defects in various MOFs and their composites. New applications of defective MOFs can be expected in fields such as optics, magnetism, sensors, photothermal therapy, electrochemical energy storage and others beyond catalysis, gas adsorption, decontamination, and drug delivery.

\section{Conflicts of interest}

There are no conflicts to declare.

\section{Acknowledgements}

The support of this work from the National Natural Science Foundation of China (No. 21536008) is gratefully acknowledged. $\mathrm{X}$. Tu acknowledges the funding from the European Union's Horizon 2020 Research and Innovation Programme under the Marie Skłodowska-Curie grant agreement No. 823745.

\section{References}

1 Y. Zheng and S.-Z. Qiao, Natl. Sci. Rev., 2018, 5, 626-627.

2 C. Tan, K. Yang, J. Dong, Y. Liu, Y. Liu, J. Jiang and Y. Cui, J. Am. Chem. Soc., 2019, 141, 17685-17695.
3 W. Xiang, S. Gebhardt, R. Gläser and C.-j. Liu, Microporous Mesoporous Mater., 2020, 300, 110152.

4 S. Chong, G. Thiele and J. Kim, Nat. Commun., 2017, 8, 1539. 5 S.-Y. Kim, A.-R. Kim, J. W. Yoon, H.-J. Kim and Y.-S. Bae, Chem. Eng. J., 2018, 335, 94-100.

6 A. Koutsianos, E. Kazimierska, A. R. Barron, M. Taddei and E. Andreoli, Dalton Trans., 2019, 48, 3349-3359.

7 H.-Y. Zhang, R.-H. Shi, H.-L. Fan, C. Yang, C.-N. Zhang, Y.-S. Wang and Z. Tian, Microporous Mesoporous Mater., 2020, 298, 110070.

8 L. M. Rodríguez-Albelo, E. López-Maya, S. Hamad, A. R. Ruiz-Salvador, S. Calero and J. A. Navarro, Nat. Commun., 2017, 8, 14457.

9 M. Erkartal and U. Sen, ACS Appl. Mater. Interfaces, 2017, 10, 787-795.

10 A. W. Thornton, R. Babarao, A. Jain, F. Trousselet and F. X. Coudert, Dalton Trans., 2016, 45, 4352-4359.

11 R. Xu, Q. Ji, P. Zhao, M. Jian, C. Xiang, C. Hu, G. Zhang, C. Tang, R. Liu, X. Zhang and J. Qu, J. Mater. Chem. A, 2020, 8, 7870-7879.

12 Y. Wang, L. Li, P. Dai, L. Yan, L. Cao, X. Gu and X. Zhao, J. Mater. Chem. A, 2017, 5, 22372-22379.

13 M. Taddei, G. M. Schukraft, M. E. A. Warwick, D. Tiana, M. J. McPherson, D. R. Jones and C. Petit, J. Mater. Chem. A, 2019, 7, 23781-23786.

14 K. L. Svane, J. K. Bristow, J. D. Gale and A. Walsh, J. Mater. Chem. A, 2018, 6, 8507-8513.

15 A. De Vos, K. Hendrickx, P. Van Der Voort, V. Van Speybroeck and K. Lejaeghere, Chem. Mater., 2017, 29, 3006-3019.

16 C. Montoro, P. Ocon, F. Zamora and J. A. Navarro, Chem.Eur. J., 2016, 22, 1646-1651.

17 R. Shimoni, W. He, I. Liberman and I. Hod, J. Phys. Chem. C, 2019, 123, 5531-5539.

18 B. Zheng, F. Fu, L. L. Wang, J. Wang, L. Du and H. Du, J. Phys. Chem. C, 2018, 122, 4300-4306.

19 S. Dissegna, P. Vervoorts, C. L. Hobday, T. Duren, D. Daisenberger, A. J. Smith, R. A. Fischer and G. Kieslich, J. Am. Chem. Soc., 2018, 140, 11581-11584.

20 C. Caratelli, J. Hajek, F. G. Cirujano, M. Waroquier, F. X. L. i Xamena and V. Van Speybroeck, J. Catal., 2017, 352, 401414.

21 Z. Jiang, L. Ge, L. Zhuang, M. Li, Z. Wang and Z. Zhu, ACS Appl. Mater. Interfaces, 2019, 11, 44300-44307.

22 G. Ye, D. Zhang, X. Li, K. Leng, W. Zhang, J. Ma, Y. Sun, W. $\mathrm{Xu}$ and S. Ma, ACS Appl. Mater. Interfaces, 2017, 9, 34937-34943.

23 X. Ma, L. Wang, Q. Zhang and H.-L. Jiang, Angew. Chem., Int. Ed., 2019, 58, 12175-12179.

24 K. Y. Cho, J. Y. Seo, H.-J. Kim, S. J. Pai, X. H. Do, H. G. Yoon, S. S. Hwang, S. S. Han and K.-Y. Baek, Appl. Catal., B, 2019, 245, 635-647.

25 G. Ye, Y. Gu, W. Zhou, W. Xu and Y. Sun, ACS Catal., 2020, 10, 2384-2394.

26 W. Wang, D. I. Sharapa, A. Chandresh, A. Nefedov, S. Heißler, L. Heinke, F. Studt and Y. Wang, Angew. Chem., Int. Ed., 2020, 59, 10514-10518. 
27 Y.-P. Xu, Z.-Q. Wang, H.-Z. Tan, K.-Q. Jing, Z.-N. Xu and G.-C. Guo, Catal. Sci. Technol., 2020, 10, 1699-1707.

28 Y. Chen, S. Zhang, F. Chen, S. Cao, Y. Cai, S. Li, H. Ma, X. Ma, P. Li, X. Huang and B. Wang, J. Mater. Chem. A, 2018, 6, 342-348.

29 J. Wang, L. Liu, C. Chen, X. Dong, Q. Wang, L. Alfilfil, M. R. AlAlouni, K. Yao, J. Huang, D. Zhang and Y. Han, J. Mater. Chem. A, 2020, 8, 4464-4472.

30 C. Hu, Y. Bai, M. Hou, Y. Wang, L. Wang, X. Cao, C.-W. Chan, H. Sun, W. Li, J. Ge and K. Ren, Sci. Adv., 2020, 6, eaax5785.

31 M. S. Alivand, M. Shafiei-Alavijeh, N. H. M. H. Tehrani, E. Ghasemy, A. Rashidi and S. Fakhraie, Microporous Mesoporous Mater., 2019, 279, 153-164.

32 X. Kang, K. Lyu, L. Li, J. Li, L. Kimberley, B. Wang, L. Liu, Y. Cheng, M. D. Frogley, S. Rudic, A. J. Ramirez-Cuesta, R. A. W. Dryfe, B. Han, S. Yang and M. Schroder, Nat. Commun., 2019, 10, 4466.

33 Z. Xue, K. Liu, Q. Liu, Y. Li, M. Li, C. Y. Su, N. Ogiwara, H. Kobayashi, H. Kitagawa, M. Liu and G. Li, Nat. Commun., 2019, 10, 5048.

34 W. Xiang, Z. Sun, Y. Wu, L.-N. He and C.-j. Liu, Catal. Today, 2020, 339, 337-343.

35 Y. Liu, R. C. Klet, J. T. Hupp and O. Farha, Chem. Commun., 2016, 52, 7806-7809.

36 G. G. Chang, X. C. Ma, Y. X. Zhang, L. Y. Wang, G. Tian, J. W. Liu, J. Wu, Z. Y. Hu, X. Y. Yang and B. Chen, Adv. Mater., 2019, 31, 1904969.

37 X. Chang, X. F. Yang, Y. Qiao, S. Wang, M. H. Zhang, J. Xu, D. H. Wang and X. H. Bu, Small, 2020, 16, 1906432.

38 F. Meng, S. Zhang, L. Ma, W. Zhang, M. Li, T. Wu, H. Li, T. Zhang, X. Lu, F. Huo and J. Lu, Adv. Mater., 2018, 30, 1803263.

39 A. M. Abdel-Mageed, B. Rungtaweevoranit, M. ParlinskaWojtan, X. Pei, O. M. Yaghi and R. J. Behm, J. Am. Chem. Soc., 2019, 141, 5201-5210.

40 G. Fu, B. Bueken and D. De Vos, Small Methods, 2018, 2, 1800203.

41 Y. Feng, Q. Chen, M. Jiang and J. Yao, Ind. Eng. Chem. Res., 2019, 58, 17646-17659.

42 J. Ren, M. Ledwaba, N. M. Musyoka, H. W. Langmi, M. Mathe, S. Liao and W. Pang, Coord. Chem. Rev., 2017, 349, 169-197.

43 M. Taddei, Coord. Chem. Rev., 2017, 343, 1-24.

44 Z. Y. Yu, Y. Duan, J. D. Liu, Y. Chen, X. K. Liu, W. Liu, T. Ma, Y. Li, X. S. Zheng, T. Yao, M. R. Gao, J. F. Zhu, B. J. Ye and S. H. Yu, Nat. Commun., 2019, 10, 2799.

45 T. Wang, H. Zhu, Q. Zeng and D. Liu, Adv. Mater. Interfaces, 2019, 6, 1900423.

46 Z. Fang, B. Bueken, D. E. De Vos and R. A. Fischer, Angew. Chem., Int. Ed., 2015, 54, 7234-7254.

47 S. Dissegna, K. Epp, W. R. Heinz, G. Kieslich and R. A. Fischer, Adv. Mater., 2018, 30, 1704501.

48 L. Liu, Z. Chen, J. Wang, D. Zhang, Y. Zhu, S. Ling, K. W. Huang, Y. Belmabkhout, K. Adil, Y. Zhang, B. Slater, M. Eddaoudi and Y. Han, Nat. Chem., 2019, 11, 622-628.
49 S. Chaemchuen, Z. Luo, K. Zhou, B. Mousavi, S. Phatanasri, M. Jaroniec and F. Verpoort, J. Catal., 2017, 354, 84-91.

50 P. Iacomi, F. Formalik, J. Marreiros, J. Shang, J. Rogacka, A. Mohmeyer, P. Behrens, R. Ameloot, B. Kuchta and P. L. Llewellyn, Chem. Mater., 2019, 31, 8413-8423.

51 G. C. Shearer, S. Chavan, S. Bordiga, S. Svelle, U. Olsbye and K. P. Lillerud, Chem. Mater., 2016, 28, 3749-3761.

52 I. Abánades Lázaro, C. J. R. Wells and R. S. Forgan, Angew. Chem., Int. Ed., 2020, 59, 5211-5217.

53 G. Cai and H. L. Jiang, Angew. Chem., Int. Ed., 2017, 56, 563567.

54 M. R. DeStefano, T. Islamoglu, S. J. Garibay, J. T. Hupp and O. K. Farha, Chem. Mater., 2017, 29, 1357-1361.

55 H. V. Doan, H. A. Hamzah, P. K. Prabhakaran, C. Petrillo and V. P. Ting, Nano-Micro Lett., 2019, 11, 54.

56 D. Liu, D. Zou, H. Zhu and J. Zhang, Small, 2018, 14, 1801454.

57 K. B. Idrees, Z. Chen, X. Zhang, M. R. Mian, R. J. Drout, T. Islamoglu and O. K. Farha, Chem. Mater., 2020, 32, 3776-3782.

58 M. Taddei, R. J. Wakeham, A. Koutsianos, E. Andreoli and A. R. Barron, Angew. Chem., Int. Ed., 2018, 57, 11706-11710.

59 F. C. N. Firth, M. J. Cliffe, D. Vulpe, M. Aragones-Anglada, P. Z. Moghadam, D. Fairen-Jimenez, B. Slater and C. P. Grey, J. Mater. Chem. A, 2019, 7, 7459-7469.

60 J. S. Lee, E. A. Kapustin, X. Pei, S. Llopis, O. M. Yaghi and F. D. Toste, Chem, 2020, 6, 142-152.

61 K. Epp, I. Luz, W. R. Heinz, A. Rapeyko, F. X. Llabrés i Xamena and R. A. Fischer, ChemCatChem, 2020, 12, 17201725.

62 Y. Gao, J. Lu, J. Xia and G. Yu, ACS Appl. Mater. Interfaces, 2020, 12, 12706-12716.

63 Z. Fan, J. Wang, W. Wang, S. Burger, Z. Wang, Y. Wang, C. Wöll, M. Cokoja and R. A. Fischer, ACS Appl. Mater. Interfaces, 2020, 12, 37993-38002.

64 S. Yuan, L. Zou, J. S. Qin, J. Li, L. Huang, L. Feng, X. Wang, M. Bosch, A. Alsalme, T. Cagin and H. C. Zhou, Nat. Commun., 2017, 8, 15356.

65 B. Bueken, N. Van Velthoven, A. Krajnc, S. Smolders, F. Taulelle, C. Mellot-Draznieks, G. Mali, T. D. Bennett and D. De Vos, Chem. Mater., 2017, 29, 10478-10486.

66 L. Feng, S. Yuan, L. L. Zhang, K. Tan, J. L. Li, A. Kirchon, L. M. Liu, P. Zhang, Y. Han, Y. J. Chabal and H. C. Zhou, J. Am. Chem. Soc., 2018, 140, 2363-2372.

67 V. Guillerm, H. Xu, J. Albalad, I. Imaz and D. Maspoch, J. Am. Chem. Soc., 2018, 140, 15022-15030.

68 K. Y. Wang, L. Feng, T. H. Yan, S. Wu, E. A. Joseph and H. C. Zhou, Angew. Chem., Int. Ed., 2020, 59, 11349-11354.

69 B. Lee, D. Moon and J. Park, Angew. Chem., Int. Ed., 2020, 59, 13793-13799.

70 C. Tan, X. Han, Z. Li, Y. Liu and Y. Cui, J. Am. Chem. Soc., 2018, 140, 16229-16236.

71 J. Koo, I. C. Hwang, X. Yu, S. Saha, Y. Kim and K. Kim, Chem. Sci., 2017, 8, 6799-6803.

72 Y. Fang, S. Banerjee, E. A. Joseph, G. S. Day, M. Bosch, J. Li, Q. Wang, H. Drake, O. K. Ozdemir and J. M. Ornstein, Chem.-Eur. J., 2018, 24, 16977-16982. 
73 H. F. Drake, G. S. Day, S. W. Vali, Z. Xiao, S. Banerjee, J. Li, E. A. Joseph, J. E. Kuszynski, Z. T. Perry and A. Kirchon, Chem. Commun., 2019, 55, 12769-12772.

74 Y. Guo, T. Wang, J. Chen, J. Zheng, X. Li and K. K. Ostrikov, Adv. Energy Mater., 2018, 8, 1800085.

75 P. Cui, P. Wang, Y. Zhao and W.-Y. Sun, Cryst. Growth Des., 2019, 19, 1454-1470.

76 S. M. Cohen, J. Am. Chem. Soc., 2017, 139, 2855-2863.

77 X. Zhang, Z. Zhang, J. Boissonnault and S. M. Cohen, Chem. Commun., 2016, 52, 8585-8588.

78 H. V. Doan, A. Sartbaeva, J. C. Eloi, S. A. Davis and V. P. Ting, Sci. Rep., 2019, 9, 10887.

79 P. Yang, F. Mao, Y. Li, Q. Zhuang and J. Gu, Chem.-Eur. J., 2018, 24, 2962-2970.

80 X. Feng, J. Hajek, H. S. Jena, G. Wang, S. K. P. Veerapandian, R. Morent, N. De Geyter, K. Leyssens, A. E. J. Hoffman, V. Meynen, C. Marquez, D. E. De Vos, V. Van Speybroeck, K. Leus and P. Van Der Voort, J. Am. Chem. Soc., 2020, 142, 3174-3183.

81 W. Xiang, J. Ren, S. Chen, C. Shen, Y. Chen, M. Zhang and C.-j. Liu, Appl. Energy, 2020, 277, 115560.

82 C.-j Liu, M. Li, J. Wang, X. Zhou, Q. Guo, J. Yan and Y. Li, Chin. J. Catal., 2016, 37, 340-348.

83 X. Zhang, Y. Yang, L. Song, J. Chen, Y. Yang and Y. Wang, J. Hazard. Mater., 2019, 365, 597-605.

84 Y. Zhao, Q. Zhang, Y. Li, R. Zhang and G. Lu, ACS Appl. Mater. Interfaces, 2017, 9, 15079-15085.

85 R. C. Klet, Y. Liu, T. C. Wang, J. T. Hupp and O. K. Farha, J. Mater. Chem. A, 2016, 4, 1479-1485.

86 W. Liang, L. Li, J. Hou, N. D. Shepherd, T. D. Bennett, D. M. D'Alessandro and V. Chen, Chem. Sci., 2018, 9, 3508-3516.

87 S. Dissegna, R. Hardian, K. Epp, G. Kieslich, M.-V. Coulet, P. Llewellyn and R. A. Fischer, CrystEngComm, 2017, 19, 4137-4141.

88 J. N. Hall and P. Bollini, Langmuir, 2020, 36, 1345-1356.

89 X. Chen, Y. Lyu, Z. Wang, X. Qiao, B. C. Gates and D. Yang, ACS Catal., 2020, 10, 2906-2914.

90 S. Smolders, T. Willhammar, A. Krajnc, K. Sentosun, M. T. Wharmby, K. A. Lomachenko, S. Bals, G. Mali, M. B. Roeffaers and D. E. De Vos, Angew. Chem., Int. Ed., 2019, 131, 9258-9263.

91 A. S. Poryvaev, D. M. Polyukhov, E. Gjuzi, F. Hoffmann, M. Fröba and M. V. Fedin, Inorg. Chem., 2019, 58, 84718479.

92 P. F. Muldoon, S. R. Venna, D. W. Gidley, J. S. Baker, L. Zhu, Z. Tong, F. Xiang, D. P. Hopkinson, S. Yi, A. K. Sekizkardes and N. L. Rosi, ACS Mater. Lett., 2020, 2, 821-828.

93 S. S. Mondal, S. Dey, A. G. Attallah, R. Krause-Rehberg, C. Janiak and H.-J. Holdt, Dalton Trans., 2017, 46, 48244833.

94 L. Yuan, M. Tian, J. Lan, X. Cao, X. Wang, Z. Chai, J. K. Gibson and W. Shi, Chem. Commun., 2018, 54, 370-373.

95 S. Waitschat, D. Fröhlich, H. Reinsch, H. Terraschke, K. A. Lomachenko, C. Lamberti, H. Kummer, T. Helling, M. Baumgartner, S. Henninger and N. Stock, Dalton Trans., 2018, 47, 1062-1070.
96 M. J. Cliffe, W. Wan, X. Zou, P. A. Chater, A. K. Kleppe, M. G. Tucker, H. Wilhelm, N. P. Funnell, F.-X. Coudert and A. L. Goodwin, Nat. Commun., 2014, 5, 4176.

97 X. Zhang, Y. Yang, X. Lv, Y. Wang, N. Liu, D. Chen and L. Cui, J. Hazard. Mater., 2019, 366, 140-150.

98 C. Li, J. Huang, H. Zhu, L. Liu, Y. Feng, G. Hu and X. Yu, Sens. Actuators, B, 2017, 253, 275-282.

99 J. Ren, N. M. Musyoka, H. W. Langmi, J. Walker, M. Mathe and S. Liao, Polyhedron, 2018, 153, 205-212.

100 F. G. Cirujano and F. X. Llabrés i Xamena, J. Phys. Chem. Lett., 2020, 11, 4879-4890.

101 D. M. Driscoll, D. Troya, P. M. Usov, A. J. Maynes, A. J. Morris and J. R. Morris, J. Phys. Chem. C, 2018, 122, 14582-14589.

102 K. Chakarova, I. Strauss, M. Mihaylov, N. Drenchev and K. Hadjiivanov, Microporous Mesoporous Mater., 2019, 281, 110-122.

103 R. Insyani, D. Verma, H. S. Cahyadi, S. M. Kim, S. K. Kim, N. Karanwal and J. Kim, Appl. Catal., B, 2019, 243, 337-354.

104 F. S. Gentile, M. Pannico, M. Causà, G. Mensitieri, G. Di Palma, G. Scherillo and P. Musto, J. Mater. Chem. A, 2020, 8, 10796-10812.

105 Y. Huang, Y. Jiao, T. Chen, Y. Gong, S. Wang, Y. Liu, D. S. Sholl and K. S. Walton, ACS Appl. Mater. Interfaces, 2020, 12, 34413-34422.

106 L. Ni, Z. Liao, K. Chen, J. Xie, Q. Li, J. Qi, X. Sun, L. Wang and J. Li, Chem. Commun., 2020, 56, 8372-8375.

107 G. Fu, F. G. Cirujano, A. Krajnc, G. Mali, M. Henrion, S. Smolders and D. E. De Vos, Catal. Sci. Technol., 2020, 10, 4002-4009.

108 R. Ameloot, F. Vermoortele, J. Hofkens, F. C. De Schryver, D. E. De Vos and M. B. Roeffaers, Angew. Chem., 2013, 125, 419-423.

109 B. M. Weckhuysen, Z. Öztürk, R. P. Brand, J. M. Boereboom and F. Meirer, Chem.-Eur. J., 2019, 25, 8070-8084.

110 W. Schrimpf, J. Jiang, Z. Ji, P. Hirschle, D. C. Lamb, O. M. Yaghi and S. Wuttke, Nat. Commun., 2018, 9, 1-10.

111 D. N. Johnstone, F. C. N. Firth, C. P. Grey, P. A. Midgley, M. J. Cliffe and S. M. Collins, J. Am. Chem. Soc., 2020, 142, 13081-13089.

112 B. Shen, X. Chen, K. Shen, H. Xiong and F. Wei, Nat. Commun., 2020, 11, 2692.

113 L. Liu, D. Zhang, Y. Zhu and Y. Han, Commun. Chem., 2020, 3, 99.

114 T. Steenhaut, N. Grégoire, G. Barozzino-Consiglio, Y. Filinchuk and S. Hermans, RSC Adv., 2020, 10, 1982219831.

115 S. Chen, Y. Zhou, J. Li, Z. Hu, F. Dong, Y. Hu, H. Wang, L. Wang, K. K. Ostrikov and Z. Wu, ACS Catal., 2020, 10, 10185-10196.

116 M. Mandal, C. J. Cramer, D. G. Truhlar, J. Sauer and L. Gagliardi, ACS Catal., 2020, 10, 10051-10059.

117 E. S. Gutterød, S. H. Pulumati, G. Kaur, A. Lazzarini, B. G. Solemsli, A. E. Gunnæs, C. Ahoba-Sam, M. E. Kalyva, J. A. Sannes, S. Svelle, E. Skulason, A. Nova and U. Olsbye, J. Am. Chem. Soc., 2020, 142, 17105-17118. 
118 Z. Zou, J. Wang, H. Pan, J. Li, K. Guo, Y. Zhao and C. Xu, J. Mater. Chem. A, 2020, 8, 14099-14105.

119 P. Ponchai, K. Adpakpang, S. Thongratkaew, K. Chaipojjana, S. Wannapaiboon, S. Siwaipram, K. Faungnawakij and S. Bureekaew, Chem. Commun., 2020, 56, 8019-8022.

120 D. Yang, C. A. Gaggioli, D. Ray, M. Babucci, L. Gagliardi and B. C. Gates, J. Am. Chem. Soc., 2020, 142, 8044-8056.

121 W. Xiang, Y. Zhang, H. Lin and C.-j. Liu, Molecules, 2017, 22, 2103.

122 M.-J. Dong, X. Wang and C.-D. Wu, Adv. Funct. Mater., 2020, 30, 1908519.

123 W. Zhang, Y. Wang, L. Ling, X. Wang, H. Chang, R. Li, W. Duan and B. Liu, Microporous Mesoporous Mater., 2020, 294, 109943.

124 E. S. Gutterod, A. Lazzarini, T. Fjermestad, G. Kaur, M. Manzoli, S. Bordiga, S. Svelle, K. P. Lillerud, E. Skulason, S. Oien-Odegaard, A. Nova and U. Olsbye, $J$. Am. Chem. Soc., 2020, 142, 999-1009.

125 P. H. Guo, Q. Fu, C. Yildiz, Y. T. Chen, K. Ollegott, C. Froese, W. Kleist, R. A. Fischer, Y. M. Wang, M. Muhler and B. X. Peng, Catal. Sci. Technol., 2019, 9, 3703-3710.

126 Z. Thiam, E. Abou-Hamad, B. Dereli, L. Liu, A.-H. Emwas, R. Ahmad, H. Jiang, A. A. Isah, P. B. Ndiaye, M. Taoufik, Y. Han, L. Cavallo, J.-M. Basset and M. Eddaoudi, J. Am. Chem. Soc., 2020, 142, 16690-16703.

127 S. Guo, Y. Zhao, C. Wang, H. Jiang and G. J. Cheng, ACS Appl. Mater. Interfaces, 2020, 12, 26068-26075.

128 J. N. Hall and P. Bollini, React. Chem. Eng., 2019, 4, 207-222. 129 Z. Ji, H. Wang, S. Canossa, S. Wuttke and O. M. Yaghi, Adv. Funct. Mater., 2020, 30, 2000238.

130 S. Ohsaki, R. Nakazawa, A. Teranishi, H. Nakamura and S. Watano, Microporous Mesoporous Mater., 2020, 302, 110215.

131 C. Jiao, Z. Majeed, G.-H. Wang and H. Jiang, J. Mater. Chem. A, 2018, 6, 17220-17226.

132 Y. Wu, H. Duan and H. Xi, Chem. Mater., 2020, 32, 29862997.

133 K. O. Kirlikovali, Z. Chen, T. Islamoglu, J. T. Hupp and O. K. Farha, ACS Appl. Mater. Interfaces, 2020, 12, 1470214720.

134 G. W. Peterson, M. R. Destefano, S. J. Garibay, A. Ploskonka, M. McEntee, M. Hall, C. J. Karwacki, J. T. Hupp and O. K. Farha, Chem.-Eur. J., 2017, 23, 15913-15916.

135 D. A. Giannakoudakis and T. J. Bandosz, ACS Appl. Mater. Interfaces, 2020, 12, 14678-14689.

136 Y. Xu, J. Lv, Y. Song, X. Zhou, C. Tian, X. Hong, Y. Cai, C. Zhao and Z. Lin, Environ. Sci.: Nano, 2019, 6, 3590-3600.
137 J.-M. Yang, B.-C. Yang, Y. Zhang, R.-N. Yang, S.-S. Ji, Q. Wang, S. Quan and R.-Z. Zhang, Microporous Mesoporous Mater., 2020, 292, 109764.

138 J. A. Chiong, J. Zhu, J. B. Bailey, M. Kalaj, R. H. Subramanian, W. Xu, S. M. Cohen and F. A. Tezcan, J. Am. Chem. Soc., 2020, 142, 6907-6912.

139 X. Zhang, X. Lv, X. Shi, Y. Yang and Y. Yang, J. Colloid Interface Sci., 2019, 539, 152-160.

140 Y. Jiao, Y. Liu, G. Zhu, J. T. Hungerford, S. Bhattacharyya, R. P. Lively, D. S. Sholl and K. S. Walton, J. Phys. Chem. C, 2017, 121, 23471-23479.

141 T. Islamoglu, M. A. Ortuño, E. Proussaloglou, A. J. Howarth, N. A. Vermeulen, A. Atilgan, A. M. Asiri, C. J. Cramer and O. K. Farha, Angew. Chem., Int. Ed., 2018, 57, 1949-1953.

142 M. R. Momeni and C. J. Cramer, ACS Appl. Mater. Interfaces, 2018, 10, 18435-18439.

143 M. R. Momeni and C. J. Cramer, Chem. Mater., 2018, 30, 4432-4439.

144 K. Wang, C. Li, Y. Liang, T. Han, H. Huang, Q. Yang, D. Liu and C. Zhong, Chem. Eng. J., 2016, 289, 486-493.

145 J. Qiu, Y. Feng, X. Zhang, M. Jia and J. Yao, J. Colloid Interface Sci., 2017, 499, 151-158.

146 P. Hu, Z. Zhao, X. Sun, Y. Muhammad, J. Li, S. Chen, C. Pang, T. Liao and Z. Zhao, Chem. Eng. J., 2019, 356, 329-340.

147 Z. Wang, S. Hu, J. Yang, A. Liang, Y. Li, Q. Zhuang and J. Gu, Adv. Funct. Mater., 2018, 28, 1707356.

148 M. H. Teplensky, M. Fantham, P. Li, T. C. Wang, J. P. Mehta, L. J. Young, P. Z. Moghadam, J. T. Hupp, O. K. Farha, C. F. Kaminski and D. Fairen-Jimenez, J. Am. Chem. Soc., 2017, 139, 7522-7532.

149 P. Yang, Y. Men, Y. Tian, Y. Cao, L. Zhang, X. Yao and W. Yang, ACS Appl. Mater. Interfaces, 2019, 11, 1120911219.

150 Z. Wang, Y. Zhang, E. C. Neyts, X. Cao, X. Zhang, B. Jang and C.-J. Liu, ACS Catal., 2018, 8, 2093-2110.

151 R. S. Forgan, Chem. Sci., 2020, 11, 4546-4562.

152 X. Zhou and C.-J. Liu, Adv. Funct. Mater., 2017, 27, 1701134.

153 Y. Fu, Z. Kang, J. Yin, W. Cao, Y. Tu, Q. Wang and X. Kong, Nano Lett., 2019, 19, 1618-1624.

154 C. Fan, R. Wang, P. Kong, X. Wang, J. Wang, X. Zhang and Z. Zheng, Catal. Commun., 2020, 140, 106002.

155 J. Ye and C.-J. Liu, Chem. Commun., 2011, 47, 2167-2169.

156 K. Xuan, Y. Pu, F. Li, J. Luo, N. Zhao and F. Xiao, Chin. J. Catal., 2019, 40, 553-566.

157 W.-G. Cui, G.-Y. Zhang, T.-L. Hu and X.-H. Bu, Coord. Chem. Rev., 2019, 387, 79-120. 